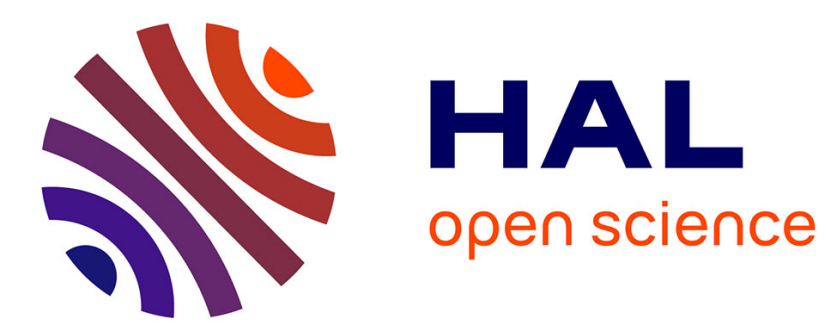

\title{
Study of interpolation methods for high-accuracy computations on overlapping grids
}

\author{
Jérémie Chicheportiche, Xavier Gloerfelt
}

\section{To cite this version:}

Jérémie Chicheportiche, Xavier Gloerfelt. accuracy computations on overlapping grids. 10.1016/j.compfluid.2012.07.019 . hal-01069633
Study of interpolation methods for highComputers and Fluids, 2012, 68, pp.112-133.

\section{HAL Id: hal-01069633}

\section{https://hal.science/hal-01069633}

Submitted on 29 Sep 2014

HAL is a multi-disciplinary open access archive for the deposit and dissemination of scientific research documents, whether they are published or not. The documents may come from teaching and research institutions in France or abroad, or from public or private research centers.
L'archive ouverte pluridisciplinaire HAL, est destinée au dépôt et à la diffusion de documents scientifiques de niveau recherche, publiés ou non, émanant des établissements d'enseignement et de recherche français ou étrangers, des laboratoires publics ou privés. 


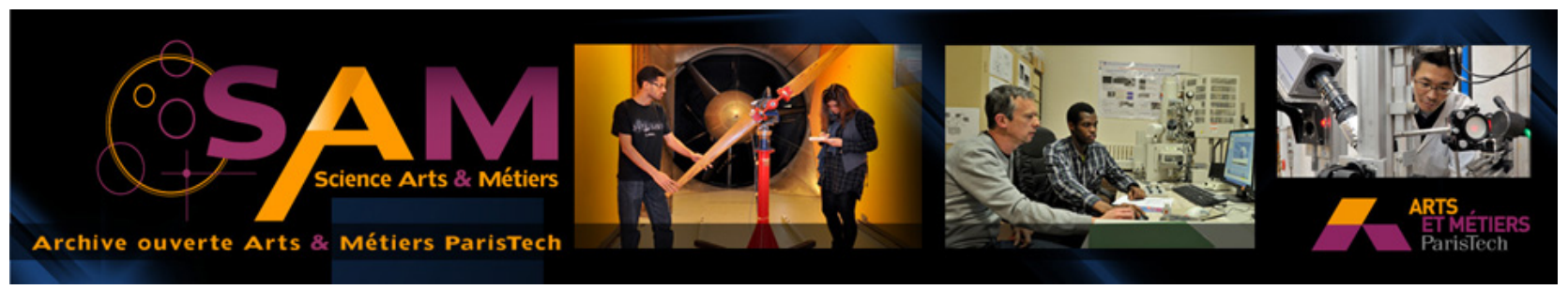

Science Arts \& Métiers (SAM)

is an open access repository that collects the work of Arts et Métiers ParisTech researchers and makes it freely available over the web where possible.

This is an author-deposited version published in: http://sam.ensam.eu

Handle ID: .http://hdl.handle.net/10985/8639

\section{To cite this version :}

Jérémie CHICHEPORTICHE, Xavier GLOERFELT - Study of interpolation methods for highaccuracy computations on overlapping grids - Computers \& Fluids - Vol. 68, p.112-133 - 2012 


\title{
Study of interpolation methods for high-accuracy computations on overlapping grids
}

\author{
J. Chicheportiche ${ }^{\mathrm{a}}$, X. Gloerfelt ${ }^{\mathrm{a}}$ \\ ${ }^{a}$ DynFluid Laboratory - Arts et Métiers ParisTech - 151 boulevard de l'Hôpital, 75013 \\ Paris, France
}

\begin{abstract}
Overset strategy can be an efficient way to keep high-accuracy discretization by decomposing a complex geometry in topologically simple subdomains. Apart from the grid assembly algorithm, the key point of overset technique lies in the interpolation processes which ensure the communications between the overlapping grids. The family of explicit Lagrange and optimized interpolation schemes is studied. The a priori interpolation error is analyzed in the Fourier space, and combined with the error of the chosen discretization to highlight the modification of the numerical error. When high-accuracy algorithms are used an optimization of the interpolation coefficients can enhance the resolvality, which can be useful when high-frequency waves or small turbulent scales need to be supported by a grid. For general curvilinear grids in more than one space dimension, a mapping in a computational space followed by a tensorization of $1-\mathrm{D}$ interpolations is preferred to a direct evaluation of the coefficient in the physical domain. A high-order extension
\end{abstract}

Email addresses: jeremie.chicheportiche@paris.ensam.fr (J. Chicheportiche), xavier.gloerfelt@paris.ensam.fr (X. Gloerfelt)

URL: www.dynfluid.eu (X. Gloerfelt) 
of the isoparametric mapping is accurate and robust since it avoids the inversion of a matrix which may be ill-conditioned. A posteriori error analyses indicate that the interpolation stencil size must be tailored to the accuracy of the discretization scheme. For well discretized wavelengthes, the results show that the choice of a stencil smaller than the stencil of the corresponding finite-difference scheme can be acceptable. Besides the gain of optimization to capture high-frequency phenomena is also underlined. Adding order contraints to the optimization allows an interesting trade-off when a large range of scales is considered. Finally, the ability of the present overset strategy to preserve accuracy is illustrated by the diffraction of an acoustic source by two cylinders, and the generation of acoustic tones in a rotor-stator interaction. Some recommandations are formulated in the closing section.

Keywords: computational aeroacoustics, overlapping grids, optimized interpolation

\section{Introduction}

Direct Noise Computation (DNC), consisting in solving the acoustic and aerodynamic fields in the same run, increasingly becomes a viable tool for analysis of engineering problems in which noise plays a significant role. In fact, DNC has already been used to study fundamental aspects of noise generation and propagation, such as jets $[1,2]$, or cavities $[3,4]$. Numerical algorithms minimizing dispersion and dissipation errors are generally required to resolve the weak acoustic wave and preserve their characteristics during long-range propagation [5]. This can be achieved by the use of high-accuracy central difference schemes [6]. Note that a similar constraint is also familiar 
in the DNS/LES framework, where fine-scale turbulent structures have to be computed on a given grid. Quasi-spectral finite-difference approximations are also widely used for that purpose due to their simplicity and efficiency. The extension to complex geometries of practical interest is however not evident. A first step has been provided by the use of curvilinear grids [7, 8] which employs body-fitted grids. This guarantees an accurate treatment of wall boundary conditions, but it is not an easy task to control the grid density: either the grid spacing deteriorates as the distance from the body increases, or the grid is too clustered in some regions, leading to a waste of computational time. Furthermore, the design of multiblock structured meshes with a sufficient regularity is often a challenging task. A solution to control the homogeneity and isotropy of the grid is the use of overset methods. This method consists in solving partial differential equations on different grids which overlap partially. The great interest is thus to decompose a complex domain into several simpler sub-domains, where the high-accuracy schemes can still be used independently. The simple sub-domains are overlapped and interpolations are used to ensure communications.

This method has been first introduced by Benek et al. [9] in the beginning of the 1980's to simulate the flow around a space shuttle. Inspired from the work of Kreiss [10], Chessire et Henshaw [11] studied the generic features of a composite grid builder. Numerous particular cases must be treated such as the overlap of two grids near a solid boundary, as explained for instance by Petersson [12]. A free library called Overture developed by Henshaw et al. [13] is now available. The next step has been to combine the overlapping grid capability with adaptive mesh refinement techniques, as demonstrated 
recently by Henshaw and Schwendeman [14], Saunier et al. [15], Sitaraman et al. [16], or Péron et al. [17]. The review by Prewitt et al. [18] shows the maturity of the method for aerodynamic applications with moving grids.

In the context of Computational AeroAcoustics (CAA), high-accuracy algorithms are generally retained, which require relatively regular grids, and large stencils to evaluate derivatives. Yin et Delfs [19] has proposed a first extension of the overset technique with Dispersion Relation Preserving (DRP) schemes [20]. Sherer and Scott [21] have developped the method for compact schemes, and Desquesnes et al. [22] have applied it to a CFD/CAA coupling. Emmert et al. [23, 24] used the Overture libraries to perform overset simulations with eleven-point stencil finite-difference DRP schemes. When high-order numerical schemes are retained, the interpolation scheme necessary to ensure the communication between the grids should not reduce the global accuracy of the algorithm.

The aim of the present paper is precisely to investigate the interpolation errors in order to choose an interpolation method tailored to the discretization algorithms. The main properties of an interpolation scheme are summarized in a first part. The family of explicit Lagrange or optimized interpolation is detailed in the second section where the extension to multidimensional state space is discussed. A static error analysis based on Fourier representation is proposed in the third section. Sensibly different conclusions can be inferred by the dynamic error analysis of the fourth section. The last section is dedicated to more challenging benchmark cases, such as the diffraction of a source by two cylinders, or the interaction of a gust and a cascade of vanes, representative of the rotor-stator interaction noise. Some 
recommendations are drawn in the concluding section.

\section{Role of interpolations for overset grids}

\subsection{Principle of the method}

The example of figure 1 provides an illustration of the principle for two overlapping grids in one space dimension. Two identical regular grids shifted by half a grid spacing are used, but the generalization to any multidimensional grids is straightforward. Grid 1 (on top) ended at index $n$, and grid 2 (bottom) starts at index 1. An information propagating from left to right is thus known on grid 1, but not on grid 2. Values must be transmitted from grid 1 to grid 2 to sustain the propagation. This communication involves interpolations from interior points (white points) of grid 1 toward interpolated points (black points) of grid 2. In overset terminology, white points are the donor points, whereas black points are receiver points, sometimes referred to as ghost points. The number of ghost points is fixed by the width of the discretization stencil. For instance, an eleven-point centered finite difference scheme is chosen in the example of figure 1, so that five ghost are added at the right of grid 1. Likewise five ghost are added at the left of grid 2 to allow a two-way communication. The region between the first interpolation point of grid 2 and the last interpolation point of grid 1 is the overlapping zone. Apart from the direct cost due to interpolation operations, the size of the overlap will add an extra cost. It is thus interesting to keep this zone to a minimum.

For explicit interpolation, the distinction between donor and receiver points imposes a minimal distance between the points, directly linked to 
the stencil of the interpolation scheme. When a donor point can also be a receiver point, the interpolation is said implicit. That means that at least one of the values used to perform the interpolation is an unknown variable to be interpolated on the other grid. The two-sided interpolation processes are coupled and require the solution of a linear system of equations, which can be expensive [11]. Nevertheless, an implicit interpolation allows a reduced overlapping zone, and becomes pertinent for complex geometries, where the gap between two bodies is small for instance. In the following of the study, only explicit interpolations are discussed. This choice is also motivated by the easier implementation on parallel computers, inevitable when three-dimensional applications on large grids are tackled.

\subsection{Properties of an interpolation scheme}

Stability issues can arise when considering non-centered stencils [25], or extrapolation [26]. It is then possible to combine an optimization in the wavenumber space and a constraint on the amplification to stabilize the interpolation scheme $[26,25]$. In the following, only centered interpolations with an even number of stencil points are considered, so that no stability issue arises.

Another issue is the conservative character of the interpolation, which is crucial for transonic or supersonic flows. Conservative interpolation schemes [27, 28, 29] are cumbersome for high-order multidimensional applications, so that practitioners prefer the use of non-conservative interpolations, which can be sufficient for weak shocks [30, 24], or in conjunction with an adaptive refinement technique to locally increase grid resolution near shocks [31, 14]. In the present paper, only subsonic compressible problems are considered. 
The main issue in the computational aeroacoustics and large-eddy simulation frameworks is the high-accuracy of the interpolation scheme. Chessire and Henshaw [11] have obtained theoretical results for elliptic problems on composite meshes, and show that the interpolation scheme should be at least of the same order as the finite-difference scheme. Delfs et al. [32] indicate that a second-order interpolation is not sufficient to propagate acoustic waves from an airfoil grid to a background Cartesian grid. The errors can be reduced by using high-order interpolation in the sense of Taylor's expansion truncation, as illustrated by Sherer and Scott [21]. These authors have tested compact approximations, which allow an enhanced resolvability. They however induce an extra cost and difficulies for parallelisation, since they are implicit (in the sense that the interpolation at a point requires the interpolated values at neighboring points). Tam et Kurbatskii [26] proposed to minimize the dissipative error in the wavenumber space rather than maximizing the Taylor truncation order, in the same spirit as the DRP optimization for finite-difference schemes [20]. Hermite interpolation is another way of increasing the accuracy, but the use of derivatives at interface points will add numerous degrees of freedom [33]. Very accurate results can also be obtained by using B-splines. An advantage is the independence of the order on the stencil width, but the imposition of constraints at end points can be tricky. Moreover a previous study for one-way communication to extrapolate nearfield acoustic waves to the far-field [4] indicated that spline interpolations are costly. Their use in multidimensional cases can become prohibitive as shown by Guénanff [33], or Sherer and Scott [21]. The discussion is hereafter restricted to explicit Lagrange or optimized interpolation schemes. 


\section{Explicit Lagrange or optimized interpolations}

\subsection{One-dimensional interpolations}

Let $N$ be the size of the interpolation stencil and $u$ a function defined on the $x_{i}$ discretization points. The explicit interpolation from $x_{i}$ points to a point $x_{0}$ is given by:

$$
u\left(x_{0}\right)=\sum_{i=1}^{N} S_{i} u\left(x_{i}\right)
$$

where $S_{i}$ are the interpolation coefficients. In this study, the coefficients are defined for centered interpolation stencils with an even number of points $N$ and the point $x_{0}$ located such that $x_{\frac{N}{2}}<x_{0}<x_{\frac{N}{2}+1}$. There are different methods to compute the coefficients.

\subsubsection{Lagrange polynomials}

The Lagrange family of interpolations has proven to be a simple and inexpensive solution $[21,34,33]$. For a stencil of size $N$, the coefficients $S_{i}$ are polynomials of degree $N-1$ defined by:

$$
S_{i}=\prod_{l=1, l \neq i}^{N} \frac{x_{0}-x_{l}}{x_{i}-x_{l}}
$$

The order of accuracy in the sense of Taylor's truncation error is $N$.

\subsubsection{Interpolations optimized in wavenumber space}

Tam and Kurbatskii [26] have computed coefficients optimized in the wavenumber space, by minimizing a local error as in the DRP procedure. The aim is to control the spectral resolvability rather than ensuring a formal order for Taylor's expansions. Compared to a classical Lagrange interpolation on the same stencil, less grid points per wavelength are required. The increased 
resolvability at small scales induces in turn a slightly reduced accuracy at very low wavenumbers. For a monochromatic wave with unitary amplitude $u_{\alpha}(x)=e^{i[k x+\phi(k)]}$, a local error in the wavenumber space can be written as:

$$
E_{\text {local }}=\left|e^{i\left(k x_{0}+\phi\right)}-\sum_{j=1}^{N} S_{j} e^{i\left(k x_{j}+\phi\right)}\right|^{2}=\left|1-\sum_{j=1}^{N} S_{j} e^{-i \frac{\left(x_{j}-x_{0}\right)}{\Delta x} k \Delta x}\right|^{2}
$$

The total error after integration over the reduced wavenumbers $k \Delta x$ between 0 and the parameter $\kappa$ writes:

$$
E=\int_{0}^{\kappa}\left|1-\sum_{j=1}^{N} S_{j} e^{-i \frac{\left(x_{j}-x_{0}\right)}{\Delta x} k \Delta x}\right|^{2} d(k \Delta x)
$$

The parameter $\kappa$ controls the range of wavenumbers over which the coefficients are optimized. Tam and Kurbatskii [26], and then Sherer and Scott [21], have shown that the error of an optimized interpolation depends on the choice of $\kappa$. Here, $\kappa$ is set to $\pi / 2$, corresponding to an optimization until 4 points per wavelength, which corresponds to the spectral limit of the discretization scheme which is used in the following. The first constraint to be imposed is that $E$ is zero for constant u, i.e. $k=0$ :

$$
E_{\text {local }}(k=0)=\left|1-\sum_{j=1}^{N} S_{j}\right|^{2}=0
$$

The minimization problem is solved with the Lagrange multiplier method to take into account the previous constraint. The Lagrangian is defined by:

$$
\mathcal{L}=\int_{0}^{\kappa}\left|1-\sum_{j=1}^{N} S_{j} e^{-i \frac{\left(x_{j}-x_{0}\right)}{\Delta x} y}\right|^{2} d y+\lambda\left(1-\sum_{j=1}^{N} S_{j}\right)^{2}
$$


with $\lambda$ the Lagrange multiplier. $S_{j}$ and $\lambda$ are then solution of the following set of equations:

$$
\begin{aligned}
\frac{\partial \mathcal{L}}{\partial S_{j}} & =0 \text { i.e. } \mathcal{R} e\left[-\int_{0}^{\kappa} e^{-i j y}\left(1-\sum_{m=1}^{N} S_{m} e^{-i \frac{x_{m}-x_{0}}{\Delta x} y}\right) d y\right]+\frac{\lambda}{2}=0 \\
\frac{\partial \mathcal{L}}{\partial \lambda} & =0 \text { i.e. } \sum_{j=1}^{N} S_{j}-1=0
\end{aligned}
$$

The explicit solution is written for instance in [26] or [21].

\subsubsection{Optimized interpolation with order constraints}

To reduce errors of an optimized interpolation at low wavenumbers, Tam and $\mathrm{Hu}[35]$ suggest to add order constraints to the Lagrangian. These order constraints come from Taylor's series expansions, and the Lagrangian then becomes:

$$
\begin{aligned}
\mathcal{L} & =\int_{0}^{\kappa}\left|1-\sum_{j=1}^{N} S_{j} e^{-i \frac{\left(x_{j}-x_{0}\right)}{\Delta x}(k \Delta x)}\right|^{2} d(k \Delta x)+\lambda\left(1-\sum_{j=1}^{N} S_{j}\right)^{2} \\
& +\sum_{m} \mu_{m}\left[\sum_{k=1}^{N} S_{k}\left(x_{k}-x_{0}\right)^{m}\right]=0
\end{aligned}
$$

where $\mu_{m}$ are the new Lagrange multipliers related to the order constraints. A new set of equations can be formed:

$$
\begin{aligned}
& \frac{\partial \mathcal{L}}{\partial S_{j}}=0 \text { i.e. } \mathcal{R} e\left[-\int_{0}^{\kappa} e^{-i j(k \Delta x)}\left(1-\sum_{m=1}^{N} S_{m} e^{-i \frac{x_{m}-x_{0}}{\Delta x}(k \Delta x)}\right) d(k \Delta x)\right]+\frac{\lambda}{2}=0 \\
& \frac{\partial \mathcal{L}}{\partial \lambda}=0 \text { i.e. } \sum_{j=1}^{N} S_{j}-1=0 \\
& \frac{\partial \mathcal{L}}{\partial \mu_{m}}=0 \text { i.e. } \sum_{k=1}^{N} m S_{k}\left(x_{k}-x_{0}\right)^{m-1}=0
\end{aligned}
$$


In the following sections, an optimized interpolation with a $m^{\text {th }}$-order constraint will mean that $m$ order constraints are added by $m$ Lagrange multipliers $\mu_{m}$.

\subsection{Two-dimensional interpolations}

Two-dimensional or three-dimensional overlapping methods can handle both Cartesian and general curvilinear non-coincident grids. The interpolation stencil varies from point to point, so that an efficient and robust algorithm to compute the interpolation coefficients at each points is required. Two different methods are presented in this section: the tensorization of 1-D interpolations, and the calculation directly in the curvilinear space. In the first method, the interpolation stencil is mapped in the regular Cartesian computational space, where 1-D interpolations can be applied by direction. The difficulty is thus reported in the choice of a particular mapping.

Let $N \times N$ be the size of the interpolation stencil, and $u$ a fonction defined on $\left(x_{i}, y_{j}\right)$ discretization points. The explicit interpolation at a point $\left(x_{0}, y_{0}\right)$ can be written as:

$$
u\left(x_{0}, y_{0}\right)=\sum_{i, j=1}^{N} S_{i j} u\left(x_{i}, y_{j}\right)
$$

where $S_{i j}$ are the interpolation coefficients.

\subsubsection{Tensorization of 1-D interpolations}

Special case of Cartesian grids. First consider the case of a $N \times N$ Cartesian interpolation stencil, as depicted in figure 2. The interpolation of $u$ at point $\left(x_{0}, y_{0}\right)$ is performed in two steps. $N$ horizontal interpolations of $u$ at abscissa $x_{0}$ for the different ordinate locations $y_{j}$ are first realized (white squares in 
figure 2). The next step is to interpolate vertically at point $\left(x_{0}, y_{0}\right)$ from the intermediate values at $\left(x_{0}, y_{j}\right)$, with $j=1, \ldots, N$. The interpolation coefficients $S_{i j}$ are thus simply the tensor products of the 1-D interpolation coefficients $S_{i}$ and $S_{j}$. For instance, for Lagrange interpolation, we get:

$$
S_{i j}=S_{i} S_{j}=\prod_{l=1, l \neq i}^{N} \frac{x_{0}-x_{l}}{x_{i}-x_{l}} \prod_{k=1, k \neq j}^{N} \frac{y_{0}-y_{k}}{y_{j}-y_{k}}
$$

The method is hereafter referred to as $2 \times 1-\mathrm{D}$ interpolation. Its extension to general curvilinear grids is based on a prior mapping of the curvilinear physical space into a Cartesian computational space, where the $2 \times 1-\mathrm{D}$ method can be readily applied. The difficulty is to determine accurately the position of the interpolated point $\left(x_{0}, y_{0}\right)$ in the reference space. This localization is straighforward if the mapping is known analytically, e.g. for conformal transformations. In particular, when a cylindrical formulation of the governing equations is used, the transformation is:

$$
\left\{\begin{array}{lll}
x=r \cos \theta & \text { and } & y=r \sin \theta \\
r=\sqrt{x^{2}+y^{2}} & \text { and } & \theta=\operatorname{atan}(y / x)
\end{array}\right.
$$

where $(x, y)$ are Cartesian coordinates and $(r, \theta)$ the polar coordinates.

Isoparametric mapping. The interpolation is realized in the computational domain $(\xi, \eta)$ as depicted in figure 3 . The location of the interpolation point is determined by the offsets $\left(\delta_{\xi}, \delta_{\eta}\right)$ relative to the base point of the stencil. The calculation of high-order offsets is performed using an isoparametric mapping from the physical space to the computational domain. The latter is Cartesian regular, so that the coefficients of a Lagrange interpolation in the 
$\alpha$-direction are defined by:

$$
S_{j}^{\alpha}=\frac{(-1)^{N+j-1}}{[N-(j+1)] ! j !} \prod_{l=0, l \neq j}^{N-1}\left(\delta_{\alpha}-j\right)
$$

Sherer and Scott [21] proposed a high-order extension of the procedure of Benek et al. [9] using explicit, non-optimized Lagrangian interpolants for the isoparametric mapping. The offsets are then solution of the following set of equations:

$$
\left\{\begin{array}{l}
F_{1}=\sum_{j=0}^{N-1} \sum_{i=0}^{N-1} S_{j}^{\eta}\left(\delta_{\eta}\right) S_{i}^{\xi}\left(\delta_{\xi}\right) x_{i, j}-x_{0}=0 \\
F_{2}=\sum_{j=0}^{N-1} \sum_{i=0}^{N-1} S_{j}^{\eta}\left(\delta_{\eta}\right) S_{i}^{\xi}\left(\delta_{\xi}\right) y_{i, j}-y_{0}=0
\end{array}\right.
$$

where $\left(x_{0}, y_{0}\right)$ and $\left(x_{i, j}, y_{i, j}\right)$ are the coordinates in the physical space for the interpolation point and the donor points forming the stencil. The interpolation coefficients $S_{i}^{\xi}$ and $S_{j}^{\eta}$ are given as functions of the offset in that direction by (7). The system is solved iteratively using Newton's method:

$$
\left[\begin{array}{c}
\delta_{\xi} \\
\delta_{\eta}
\end{array}\right]^{n+1}=\left[\begin{array}{c}
\delta_{\xi} \\
\delta_{\eta}
\end{array}\right]^{n}-\left[\mathbf{J}^{-1} \times\left(\begin{array}{c}
F_{1} \\
F_{2}
\end{array}\right)\right]^{n}
$$

where the inverse of the Jacobian matrix $\mathbf{J}$ is given by:

$$
\mathbf{J}^{-1}=\frac{1}{D}\left[\begin{array}{cc}
\frac{\partial F_{2}}{\partial \delta_{\eta}} & -\frac{\partial F_{1}}{\partial \delta_{\eta}} \\
-\frac{\partial F_{2}}{\partial \delta_{\xi}} & \frac{\partial F_{1}}{\partial \delta_{\xi}}
\end{array}\right] \text { with } D=\frac{\partial F_{1}}{\partial \delta_{\xi}} \frac{\partial F_{2}}{\partial \delta_{\eta}}-\frac{\partial F_{1}}{\partial \delta_{\eta}} \frac{\partial F_{2}}{\partial \delta_{\xi}}
$$

The derivatives can be expressed as:

$$
\frac{\partial F_{j}}{\partial \delta_{\alpha}}=\frac{(-1)^{N+j-1}}{[N-(j+1)] ! j !} \sum_{l=0, l \neq j}^{N-1} \prod_{k=0, k \neq j, k \neq l}^{N-1}\left(\delta_{\alpha}-k\right)
$$

The initial guess for the offsets $\left(\delta_{\xi}, \delta_{\eta}\right)$ is provided by the second-order offsets calculated by the grid-assembly program Overture [13]. 
Polynomial approximation of the inverse mapping. Another approach, as proposed by Guénanff et al. [36, 33], and later used by Desquesnes et al. [22], consists in defining a polynomial approximation $\mathcal{P}_{H}$ of the inverse mapping $H$, such that $H(x, y)=(\xi, \eta)$. The location of the interpolation point in the computational space is then directly determined by evaluating the polynomial $\mathcal{P}_{\mathcal{H}}$ at $\left(x_{0}, y_{0}\right)$. The determination of the polynomial coefficient requires the inversion of a matrix, which can be ill-conditioned for large stencils and particular orientations of the donor grid [33].

\subsubsection{2-D interpolations in a curvilinear space}

Another possibility is to compute the interpolation coefficients directly in the physical space. The system of Taylor's expansion relationships and/or optimization constraints is written and solved in the physical space. The general formula for a 2-D interpolation $u$ at the point $\left(x_{0}, y_{0}\right)$ is now expressed as:

$$
u\left(x_{0}, y_{0}\right)=\sum_{k=1}^{N \times N} S_{k} u\left(x_{k}, y_{k}\right)
$$

where $S_{k}$ are the interpolation coefficients to be determined.

Multidimensional interpolation with order contraints. The Taylor series expansion of order $M$ of the function $u$ at the donor points $\left(x_{k}, y_{k}\right)$ about $\left(x_{0}, y_{0}\right)$ reads:

$$
u\left(x_{k}, y_{k}\right)=u\left(x_{0}, y_{0}\right)+\sum_{p, q} \frac{1}{p ! q !}\left(\frac{\partial^{p+q} u}{\partial x^{p} \partial y^{q}}\right)_{x_{0}, y_{0}}\left(x_{k}-x_{0}\right)^{p}\left(y_{k}-y_{0}\right)^{q},
$$

for $1 \leqslant p+q \leqslant M$. The system of equations for the coefficients $S_{k}$ is obtained by replacing $u\left(x_{k}, y_{k}\right)$ by its expansion (10) in (9), and by canceling all the 
monomial terms up to order $M$. This yields:

$$
\sum_{k=1}^{N} S_{k}=1, \text { and } \sum_{k=1}^{N} S_{k}\left(x_{k}-x_{0}\right)^{p}\left(y_{k}-y_{0}\right)^{q}=0, \quad 1 \leqslant p+q \leqslant M
$$

which can be written in the matrix form:

$$
\mathbf{C S}=\left(\begin{array}{lllll}
1 & 0 & \ldots & 0 & 0
\end{array}\right)^{\mathrm{T}}
$$

The size of $\mathbf{C}$ is $T \times N$ with:

$$
T=1 / 2(M+1)(M+2), \quad C_{p q, k}=\left(x_{k}-x_{0}\right)^{p}\left(y_{k}-y_{0}\right)^{q},
$$

and $\mathbf{S}=\left(\begin{array}{llll}S_{1} & S_{2} & \ldots & S_{N}\end{array}\right)^{\mathrm{T}}$ is the unknown vector of coefficients.

Multidimensional interpolation optimized in wavenumber space. Tam and $\mathrm{Hu}$ [35] have proposed a multidimensional extension of the optimized 1-D interpolation of Tam and Kurbatskii [26]. The 2-D inverse Fourier transform is defined as:

$$
u(x, y)=\iint_{-\infty}^{+\infty} A\left(k_{1}, k_{2}\right) e^{i\left(k_{1} x+k_{2} y+\phi_{k_{1}} k_{2}\right)} d k_{1} d k_{2}
$$

with $A\left(k_{1}, k_{2}\right)=\left|\tilde{u}\left(k_{1}, k_{2}\right)\right|, \phi_{k_{1}, k_{2}}=\arg \left[\tilde{u}\left(k_{1}, k_{2}\right)\right]$. $\tilde{u}$ denotes the Fourier transform of $u$. For a unitary amplitude simple wave $u_{k_{1} k_{2}}=e^{i\left(k_{1} x+k_{2} y+\phi_{k_{1} k_{2}}\right)}$, the local error is given by:

$$
E_{\text {local }}\left(k_{1}, k_{2}\right)=\left|1-\sum_{k=1}^{N} S_{k} e^{i\left(k_{1} \Delta_{x}\left(x_{k}-x_{0}\right) / \Delta_{x}+k_{2} \Delta_{y}\left(y_{k}-y_{0}\right) / \Delta_{y}\right)}\right|
$$

Initially, for a cloud of points, Tam and $\mathrm{Hu}$ [35] introduced a unique length scale $\Delta$ to form the reduced wavenumber, which is the mean distance between the different points. Le Garrec et al. [37] proposed for structured meshes 
to distinguish mean spacings in each direction, $\Delta_{x}$ and $\Delta_{y}$, as expressed in equation (14). This trick is useful to treat flattened cells. The reduced wavenumbers are then noted $\alpha=k_{1} \Delta_{x}$, and $\beta=k_{2} \Delta_{y}$. The integrated interpolation error $E$,

$$
E=\iint_{-\kappa}^{+\kappa}\left|1-\sum_{k=1}^{N} S_{k} e^{i\left(\alpha\left(x_{k}-x_{0}\right) / \Delta_{x}+\beta\left(y_{k}-y_{0}\right) / \Delta_{y}\right)}\right|^{2} d \alpha d \beta
$$

is minimized over $-\kappa \leqslant \alpha, \beta \leqslant \kappa$, where $\kappa$ represents the highest wavenumber considered. The error must be zero for a constant function, i.e. $\sum_{k=1}^{N} S_{k}=1$, using Lagrange multiplier technique. The Lagrangian then reads:

$$
\mathcal{L}=\iint_{-\kappa}^{+\kappa}\left|1-\sum_{k=1}^{N} S_{k} e^{i\left(\alpha\left(x_{k}-x_{0}\right) / \Delta_{x}+\beta\left(y_{k}-y_{0}\right) / \Delta_{y}\right)}\right|^{2} d \alpha d \beta+\lambda\left(\sum_{k=1}^{N} S_{k}-1\right)
$$

where $\lambda$ is the Lagrange multiplier. The minimization implies:

$$
\frac{\partial \mathcal{L}}{\partial S_{k}}=0, \quad k=1,2, \ldots, N \quad \text { and } \quad \frac{\partial \mathcal{L}}{\partial \lambda}=0
$$

which can be written in the matrix form $\mathbf{A} \mathbf{S}=\mathbf{b}$, with:

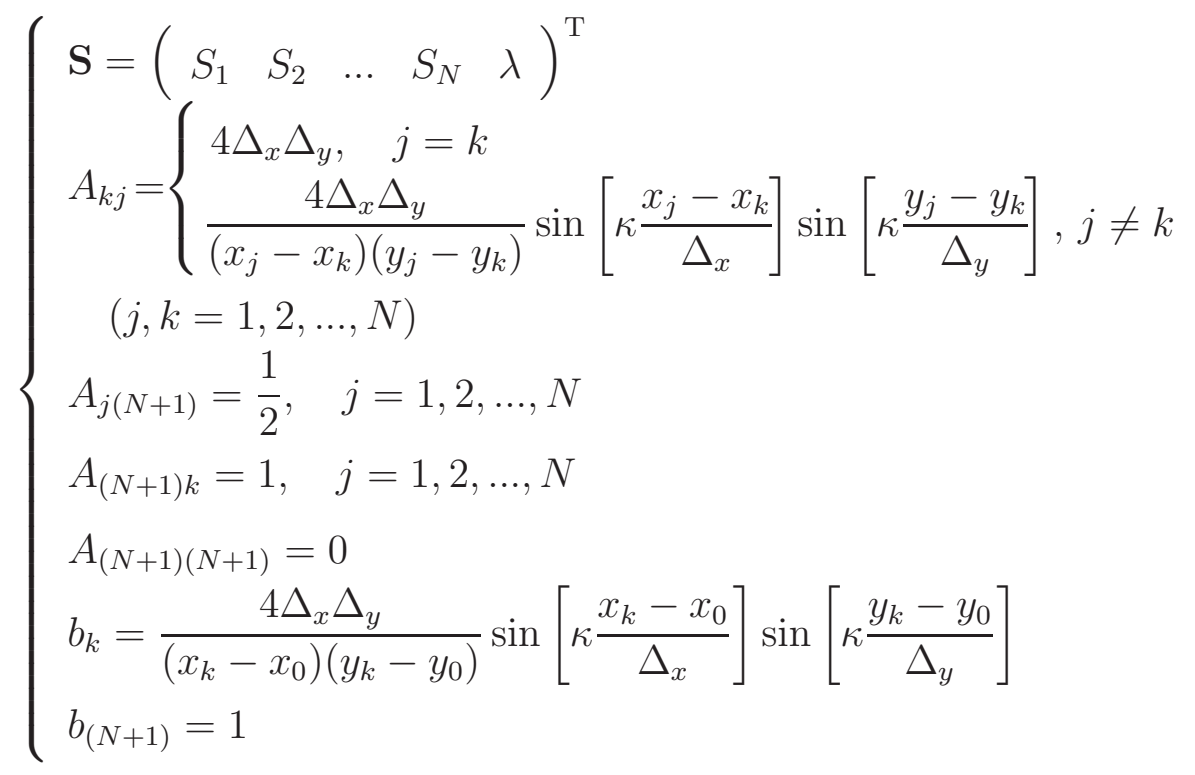


Multidimensional optimization in wavenumber space and order constraints. The enhanced resolvability due to the optimization process is at the price of a reduction of the accuracy at low wavenumbers with respect to a standard scheme. A good tradeoff can be assessed by adding order constraints, corresponding to the Taylor's expansion relationships, in the previous Lagrangian:

$$
\begin{aligned}
\mathcal{L}=\iint_{-\kappa}^{+\kappa} & \left|1-\sum_{k=1}^{N} S_{k} e^{i\left(\alpha\left(x_{k}-x_{0}\right) / \Delta_{x}+\beta\left(y_{k}-y_{0}\right) / \Delta_{y}\right)}\right|^{2} d \alpha d \beta \\
& +\lambda\left(\sum_{k=1}^{N} S_{k}-1\right)+\sum_{n, m} \mu_{m n}\left[\sum_{k=1}^{N} S_{k}\left(x-x_{0}\right)^{n}\left(y-y_{0}\right)^{m}\right]=0,
\end{aligned}
$$

where $\mu_{m n}$ are the new Lagrange multipliers. To have a minimum, we need :

$$
\frac{\partial \mathcal{L}}{\partial S_{k}}=0, \quad \frac{\partial \mathcal{L}}{\partial \lambda}=0 \quad \text { and } \quad \frac{\partial \mathcal{L}}{\partial \mu_{m n}}
$$

yielding the linear system $\mathbf{B} \mathbf{S}=\mathbf{d}$ with

$$
\begin{aligned}
& \mathbf{S}=\left(S_{1} S_{2} \ldots S_{N} \lambda \mu_{10} \mu_{01} \mu_{20} \mu_{11} \mu_{02} \ldots \mu_{m n} \ldots \mu_{0 M}\right)^{\mathrm{T}} \\
& \mathbf{d}=\left(\begin{array}{lllllll}
b_{1} & \ldots & b_{N} & 1 & 0 & \ldots & 0
\end{array}\right)^{\mathrm{T}},
\end{aligned}
$$

and

$$
\mathbf{B}=\left[\begin{array}{ll}
\mathbf{A} & \mathbf{C}^{\mathrm{T}} \\
\mathrm{C} & \mathbf{0}
\end{array}\right]
$$

$\mathbf{A}$ is given in the previous section, and the matrix $\mathbf{C}$ of size $(T-1) \times(N+1)$ is defined by:

$$
\left\{\begin{array}{l}
C_{m n, k}=\left(x-x_{0}\right)^{n}\left(y-y_{0}\right)^{m}, \quad k=1,2, \ldots, N \\
C_{m n, N+1}=0
\end{array}\right.
$$




\section{Static error analysis}

\subsection{Spectral analysis of 1-D interpolation schemes}

The square root of the local interpolation error, defined by equation (3), is plotted as a function of the reduced wavenumber $k \Delta x$. The stencil size is varied between 2 and 12, and the coefficients are computed using the different methods with or without optimization. The interpolation point is located at the center of the stencil. Sherer and Scott [21] have indeed shown that the upper bound of the error is obtained for the mid-point interpolation.

First the local error is plotted in figure 4 for the Lagrange interpolation, i.e. without optimization. The error is seen to decrease as the stencil size is augmented. However this decrease is not uniform, and important errors are still present at high wavenumbers. For instance, if the error at $\pi / 4$ with a 12-point stencil is four orders of magnitude lower than with a 4-point stencil, the gain is only one order of magnitude at $\pi / 2$.

Figure 5 presents the interpolation error for the fully optimized coefficients. As the stencil width is increased, the error diminishes, and the resolvability is enhanced. The significant improvements at high wavenumbers are counterbalanced by greater levels of error at low wavenumbers. The number of the "bounces" associated with optimization increases for the larger stencils while their amplitude is reduced. Beyond a stencil size of $N=6$, the highest resolved wavenumber is close to the imposed optimization limit $\kappa=\pi / 2$. The error level decreases globally over the wavenumbers between 0 and $\pi / 2$, so that the error can be controlled over a large range of scales. 
The error in the wavenumber space for the Lagrange and optimized interpolations are compared in figure 6. The stencil width is 8 . The gain of the optimization process is clearly visible for wavenumbers greater than $\pi / 4$ up to the limit of $\pi / 2$. The residual error levels are however higher at low wavenumbers.

Figure 7 shows the local error for optimized interpolations with order constraints, and a fixed stencil $N=8$. The addition of order constraints is seen to improve the low wavenumber range. But in the same time, the error increases for wavenumbers greater than $k \Delta x=\pi / 4$. A kind of average between the behaviour of Lagrange and fully optimized interpolation is obtained. When $8^{\text {th }}$-order constraint are added, we retrieve exactly the $8^{\text {th }}$-order Lagrange interpolation, which is superimposed in figure 7. The choice of the level of order constraints to add will be guided by the necessity or not to represent small scales on a given grid.

\subsection{Error by combining interpolation and discretization scheme}

In fact, the accuracy of the interpolation scheme should be compatible with the choice of a particular discretization scheme. In this section, the effect of the interpolation error on the resolvability of the discretization scheme is investigated. The spatial derivative is approximated by an eleven-point centered finite-difference scheme, which is part of the Euler solver presented in section 5.1. As in the 1-D example discussed in the first section, 5 ghost points are required, so that at most 5 interpolated values are used to evaluate a derivative in the overlapping zone. The interpolation formula is now written 
as:

$$
f\left(x_{0}\right)=\sum_{l=-N}^{N} S_{l} f\left(x_{0}+l\left(1-\frac{\eta}{|l|}\right) \Delta x\right)
$$

with $\eta=0.5$, the offset between the donor and receiver grids. The interpolation points are thus at the center of the interpolation stencil. The finitedifference derivative reads:

$$
\frac{\partial f}{\partial x}\left(x_{0}\right)=\frac{1}{\Delta x} \sum_{j=-5}^{5} a_{j}^{11} f\left(x_{0}+j \Delta x\right)
$$

The coefficients $a_{j}^{11}$, given in [38], are optimized by a DRP method, and correspond to a fourth-order scheme on an eleven-point stencil. Consider that only the $q \leq 5$ first points of the derivative stencil are interpolated before the derivation. Using (21) in (22) yields:

$$
\begin{aligned}
\frac{\partial f}{\partial x}\left(x_{0}\right)=\frac{1}{\Delta x}[ & \sum_{j=-5}^{q} a_{j}^{11} \sum_{l=-N}^{N} S_{l} f\left(x_{0}+\left(l\left(1-\frac{\eta}{|l|}\right)+j\right) \Delta x\right) \\
& \left.+\sum_{j=q+1}^{5} a_{j}^{11} f\left(x_{0}+j \Delta x\right)\right]
\end{aligned}
$$

For a simple wave $f_{k}=e^{i k x}$, the effective wavenumber of the scheme combining the derivative and $q$ interpolations is given by:

$$
k^{*} \Delta x=-i\left[\sum_{j=-5}^{q} a_{j}^{11} e^{i k j \Delta x} \sum_{l=-N}^{N} S_{l} e^{i k\left(1-\frac{\eta}{|l|}\right) \Delta x}+\sum_{j=q+1}^{5} a_{j}^{11} e^{i k j \Delta x}\right]
$$

Figures 8 to 13 present the dispersion error of a scheme combining the eleven-point derivative and $q$ interpolations, with $1 \leq q \leq 5$. First Lagrange interpolations are used on a stencil of size $N=8$ in figure 8 . The error for the finite-difference alone will serve as a target. The errors coming from the interpolations should indeed not reduce the overall accuracy. The added 
dispersion error remains acceptable when one or two values are interpolated beforehand ( $q=1$ and $q=2$ ). For more than three interpolated points $(q \geq 3)$, the error is increased for the wavenumbers between $\pi / 4$ and $\pi / 2$. The drop at low wavenumbers is however almost not modified. Figure 9 depicts the same results when a fully optimized interpolation is retained. When 1 to 4 interpolated points are used, the global level of error is preserved, except at very low wavenumbers. For $q=5$, the imposed limit of resolvability $\kappa=\pi / 2$ is still effective, but the error is uniformly increased in the range $[0 ; \pi / 2]$.

As in the previous Fourier analysis, the addition of order constraints improves the behaviour at low wavenumbers, as seen in figures 10 and 11, where fourth-order and sixth-order constraint are added respectively. The stencil size is the same as previously $(N=8)$. The deterioration in the intermediate wavenumbers is more pronounced with sixth-order constraint. The resolvability limit is also slightly shifted below $\pi / 2$ for $q=5$. The best compromise for a $N=8$ stencil is thus obtained with optimized interpolation and fourthorder constraints. Now the stencil size is varied for this method. Figure 12 shows the dispersion error for optimized interpolation with fourth-order constraints on a $N=10$ stencil, i.e. the same width as the finite-difference stencil. Error levels are weakly affected by the interpolation processes. An enlargement of the stencil at $N=12$ does not provide significant improvements, as seen in figure 13. The interpolation errors are now smaller than the dispersion error due the derivative over the full range of wavenumbers.

\subsection{Spectral analysis of 2-D interpolation methods}

A two-dimensional Fourier analysis is conducted to investigate the a priori error of multidimensional interpolations. The stencil used in this part is 
depicted in figure 14 . The $8 \times 8$ stencil is taken from a polar grid. Its main orientation is $45^{\circ}$ from the horizontal axis, which is a disadvantageous configuration as shown for instance in $[33,39]$. The stencil is hereafter treated as issued from a general curvilinear grid. The use of this particular polar arrangement allows the use of the analytical mapping (6). The error for $2 \times 1$-D interpolation with the analytical mapping is thereby plotted as a reference in the figures.

Like in the one-dimensional analysis, the interpolated point is at the center of the stencil, with respect to $(r, \theta)$ coordinates. The local interpolation error is provided by equation (14), where the length scales $\Delta_{x}$ and $\Delta_{y}$ correspond to the radial and azimutal spacings respectively.

Effect of the choice of the mapping for $2 \times 1-D$ interpolations. First the influence of the coordinate mapping for a curvilinear stencil is investigated. A $2 \times 1$-D Lagrange interpolation is performed after the mapping. Figure 15(a) shows isocontours of the error using a polynomial approximation of the inverse mapping. No differences are visible when compared to the analytic mapping (polar transform). On the profiles plotted in figure 15(b), small discrepancies are noticeable at very low wavenumbers. They can be due to spurious noise induced by the inversion of the polynomial mapping matrix. Guénanff [33] has indeed shown that this matrix can be ill-conditioned, notably when the main orientation of the curvilinear stencil is near $45^{\circ}$. The error profiles for the isoparametric mapping (8) are plotted in figure 15(c), and are superimposed on those with the analytic mapping. In that case, the Newton's iterations allow arbitrarily accurate convergence. 
Interpolation in the curvilinear space. The same stencil is used to test the multidimensional interpolations, whose coeffcients are directly computed in the physical space. Figure 16(a) compares the standard multidimensional interpolation (i.e. without optimization) with the reference $2 \times 1-\mathrm{D}$ Lagrange interpolation with the polar mapping. The isocontours are flattened indicating levels of error increased by a factor of ten in the $k_{2}$-direction. The profiles in the $k_{1}$-direction is close to the reference, as seen in figure 16(b). The error growth is visible on the profile in the diagonal direction. In fact, the condition number of the matrix $\mathbf{C}$ in (12) increases when large stencils are used. For the $8 \times 8$ stencil, the matrix is close to singular. Le Garrec et al. [37] showed that optimization can remove partially the singular character of the problem. In the present case, the optimized version of the multidimensional interpolation also leads to an ill-conditioned system, and the error is high on the profiles shown in figure 16(c). Adding order constraint to the optimization does not solve the problem, since an intermediate result is expected. Finally the calculation of the interpolation coefficients in the physical space is hardly achievable when large stencil are used, so that the multidimensional interpolation is not retained in the following.

Optimized $2 \times 1 D$ interpolation with or without order constraints. The eventual gains due to the optimization for the $2 \times 1-\mathrm{D}$ interpolation are investigated. The isoparametric mapping is used since it yields similar results as the analytic mapping. Isocontours of the error in the 2-D wavenumber space are depicted in figure 17 (a) for the fully optimized $2 \times 1-\mathrm{D}$ interpolation. The regions of low error levels are extended compared to the Lagrange version, notably in the diagonal directions. The lobes correspond to the "bounces", 
visible on the profiles in figure $17(\mathrm{~b})$. The profile in the $k_{1}$-direction is incidentally very close to its one-dimensional counterpart, plotted in figure 6 . The conclusions are thus the same as in the 1-D analysis: the resolvability is enhanced at the price of higher error levels for low wavenumbers. Adding fourth-order or sixth-order constraint to the optimization allows a trade-off between the low and high wavenumber ranges, as shown in figure 17(c).

To sum up for a general curvilinear stencil, the isoparametric mapping is robust and allows a simple $2 \times 1 \mathrm{D}$ interpolation in the computational space. Working directly in the physical space often lead to ill-conditioned systems for the interpolation coefficients. Within the framework of $2 \times 1 \mathrm{D}$ interpolations, the choice of an optimization in the wavenumber space is dictated by the presence of small scales, such as high-frequency acoustic waves or fine turbulent scales. A convenient trade-off is obtained by adding order-constraint, as already underlined in the 1-D analysis.

\section{Dynamic error analysis}

In order to investigate the complex interactions between the interpolation procedure and the discretization algorithm, a posteriori analyses can be conducted on simple cases for which analytical solutions are known. In particular the role of the overlapping zone, where the solution coexists on two grids, can be studied. Since the performance of the interpolation is measured in regard to the expected accuracy of the flow solver, we first present briefly the discretization retained in the study. 


\subsection{Presentation of the flow solver}

\subsubsection{Governing equations}

The governing equations are the Euler equations. Three solvers are coupled solving the Cartesian, polar and curvilinear formulations:

Cartesian formulation.

$$
\frac{\partial \mathbf{U}}{\partial t}+\frac{\partial \mathbf{E}}{\partial x}+\frac{\partial \mathbf{F}}{\partial y}=\frac{\partial S}{\partial t}
$$

where $\mathbf{U}=(\rho, \rho u, \rho v, \rho E)^{\mathrm{T}}$ is the unknow vector of conservative variables. $\rho, p$, and $(u, v)$ are the density, the pressure and the horizontal and vertical velocity components. The convective fluxes are:

$$
\begin{aligned}
& \mathbf{E}=\left(\rho u, p+\rho u^{2}, \rho u v,(\rho E+p) u\right)^{\mathrm{T}} \\
& \mathbf{F}=\left(\rho v, \rho v u, p+\rho v^{2},(\rho E+p) v\right)^{\mathrm{T}}
\end{aligned}
$$

$E$ is the total energy defined by:

$$
E=p /[(\gamma-1) \rho]+\left(u^{2}+v^{2}\right) / 2
$$

The system is closed by the ideal gas law $p=\rho r T$, where $r$ is the gas constant, and $\gamma$ is the ratio of specific heats. $S$ in (24) represents a source term.

Polar formulation.

$$
\frac{\partial \mathbf{U}}{\partial t}+\frac{1}{r} \frac{\partial(r \mathbf{E})}{\partial r}+\frac{1}{r} \frac{\partial \mathbf{F}}{\partial \theta}+\frac{\mathbf{B}}{r}=\frac{\partial S}{\partial t}
$$

with the unknow vector $\mathbf{U}=\left(\rho, \rho u_{r}, \rho u_{\theta}, \rho E\right)^{\mathrm{T}}$, and:

$$
\begin{aligned}
& \mathbf{E}=\left(\rho u_{r}, p+\rho u_{r}^{2}, \rho u_{r} u_{\theta},(\rho E+p) u_{r}\right)^{\mathrm{T}} \\
& \mathbf{F}=\left(\rho u_{\theta}, \rho u_{\theta} u_{r}, p+\rho u_{\theta}^{2},(\rho E+p) u_{\theta}\right)^{\mathrm{T}} \\
& \mathbf{B}=\left(0,-\left(\rho u_{\theta}^{2}+p\right), \rho u_{r} u_{\theta}, 0\right)^{\mathrm{T}}
\end{aligned}
$$


$u_{r}$ and $u_{\theta}$ are the radial and azimutal velocity components, and the internal energy is given by:

$$
E=p /[(\gamma-1) \rho]+\left(u_{r}^{2}+u_{\theta}^{2}\right) / 2
$$

Curvilinear formulation. A coordinate transform is used. Let $(\xi, \eta)$ be the coordinates in the computational space, the set of equations (24) becomes:

$$
\frac{\partial \mathbf{U}}{\partial t}+\frac{\partial \mathbf{F}_{\mathbf{c}}}{\partial \xi}+\frac{\partial \mathbf{G}_{\mathbf{c}}}{\partial \eta}=0
$$

by defining the curvilinear fluxes as:

$$
\left\{\begin{array}{l}
\mathbf{F}_{\mathbf{c}}=\mathbf{F} \frac{\partial \xi}{\partial x}+\mathbf{G} \frac{\partial \xi}{\partial y} \\
\mathbf{G}_{\mathbf{c}}=\mathbf{F} \frac{\partial \eta}{\partial x}+\mathbf{G} \frac{\partial \eta}{\partial y}
\end{array}\right.
$$

where $\mathbf{F}$ and $\mathbf{G}$ are given by (25). The following relationships are then used:

$$
\frac{\partial \xi}{\partial x}=\frac{1}{J} \frac{\partial y}{\partial \eta}, \quad \frac{\partial \xi}{\partial y}=-\frac{1}{J} \frac{\partial x}{\partial \eta}, \quad \frac{\partial \eta}{\partial x}=-\frac{1}{J} \frac{\partial y}{\partial \xi}, \quad \frac{\partial \eta}{\partial y}=\frac{1}{J} \frac{\partial x}{\partial \xi},
$$

where $J$ is the Jacobian:

$$
J=\left|\begin{array}{ll}
\frac{\partial x}{\partial \xi} & \frac{\partial y}{\partial \xi} \\
\frac{\partial x}{\partial \eta} & \frac{\partial y}{\partial \eta}
\end{array}\right|
$$

\subsubsection{Numerical methods}

The spatial discretization requires schemes that minimize the dispersion and the dissipation errors. Centered finite-difference schemes, intrinsically non dissipative and optimized in the wavenumber space in order to have good spectral properties, have been proposed [20]. Here, the explicit finitedifference scheme with an eleven-point stencil, optimised by Bogey and Bailly [38], is used. The expression of a spatial derivative is given by equation (22). 
By applying a spatial Fourier transform, the error between the effective and the exact wavenumbers, $k^{*} \Delta x$ and $k \Delta x$, is obtained in figure 18. The logarithmic scale of figure 18(b) shows clearly that the dispersion is maintained at a very low level up to the limit of resolvability $k \Delta x=\pi / 2$, corresponding to four points per wavelength. Grid-to-grid oscillations $(k \Delta x=\pi$ i.e. two points per wavelength, the Nyquist limit) are not resolved, so that they must be eliminated. We use a centered filter built on an eleven-point stencil [38], incorporated in each direction. For the direction $x$, the filtered quantity $f^{f}$ is computed as:

$$
f^{f}\left(x_{0}\right)=f\left(x_{0}\right)-\chi D_{f}\left(x_{0}\right) \quad \text { with } \quad D_{f}\left(x_{0}\right)=\sum_{j=-5}^{5} d_{j}^{11} f\left(x_{0}+j \Delta x\right),
$$

with the coefficient $\chi$ fixed to 0.2 . The filter has symmetric coefficients $d_{j}^{11}$, so that it is non-dispersive, and the dissipation error is minimized in the wavenumber space up to $k \Delta x=\pi / 2$. Its transfer function is superimposed in figure 18.

The governing equations are integrated in time using an explicit lowstorage six-step Runge-Kutta scheme [38], whose dispersion and dissipation errors have been minimized in frequency space up to $\omega \Delta t=\pi / 2$, corresponding to spatial fluctuations at $k \Delta x=\pi / 2$ (with $\Delta t=\operatorname{CFL} \Delta x / c_{\infty}$, and $\mathrm{CFL}=1$ ), see figure 19. Fluctuations with four points per wavelength are thus resolved spatially and temporally.

\subsubsection{Overset strategy}

A set of overset grids consists in overlapping grids with different order of priority. The grids with the higher priorities are the one which are locally the 
most adapted to the geometry of the computational domain. In most applications, these grids are body-fitted grids. The grids with the lower priorities compose the background grids. They are mostly Cartesian grids and are sized on the dimension of the computational domain. The background grids are overlapped by the smaller grids with the high priorities where the numerical solution does not have to be computed and a hole is then created: it is a cutting hole process. The cutting hole must have the appropriate size to ensure a sufficient overlapping and to avoid the computation at useless points of the background grids. For complex geometries, the algorithm related to cutting hole process is difficult to implement [11]. In this study, the grid generator Ogen from the free library Overture is used to generate overlapping grids [13]. This grid generator realizes cutting hole processes and provides us the connectivity list between ghost points of a grid and stencil points from an other grid. Since an eleven-point stencil is used to evaluate first-order derivatives and to filter spurious solutions, five ghost points are added to the interpolation interfaces of each grids. The coefficients of interpolation on the ghost points are computed in a pre-processing step. Interpolations are performed once at each sub-step of the Runge-Kutta scheme, and once after filtering the solution at the last sub-step of the Runge-Kutta algorithm.

\subsection{Advection of a 1-D wavepacket}

A preliminary one-dimensional test is performed. A simple linear advection equation is solved:

$$
\frac{\partial w}{\partial t}+\frac{\partial w}{\partial x}=0
$$

with the algorithm presented in the previous section. The extent of the grid can be inferred from figure 20. The central part is overlapped by a grid 
shifted by half a grid spacing. Its length is one third of the total length, and both grids are regular with $\Delta x=1$. The initial condition is a wavepacket defined by:

$$
w(x)=\sin \left(\frac{2 \pi x}{a \Delta x}\right) \exp \left(-\ln 2\left(\frac{x}{b \Delta x}\right)^{2}\right)
$$

where $a \Delta x$ is the disturbance wavelength, and $b \Delta x$ represents the half-width of the Gaussian wavepacket. We set $a=5$ and $b=9$, corresponding to 5 points per wavelength (close to the resolvability limit of the discretization schemes). The packet is initially located at $x=0$ as seen in figure 20 , and is advected over a distance $N_{i t} \Delta x$, with the number of iterations $N_{i t}=200$. The packet has then gone through the overset insert, and a mean quadratic error is computed as:

$$
L=\frac{1}{N_{i t}} \sum_{n=1}^{N_{i t}}\left(\sqrt{\frac{\sum_{i}\left(w_{i}-w_{\text {exact }}\right)^{2}}{\sum_{i} w_{\text {exact }}^{2}}}\right)_{n}
$$

The error normalized by the error of the background grid alone is plotted in figure 21 by varying systematically the interpolation stencil size, and the method to compute interpolation coefficients. Of course, the width of the overlap depends on the stencil size, but the error is evaluated only for noninterpolated point, so that this difference is not important.

As the size of the interpolation stencil is increased, the error is reduced and tends toward the value without overlapping. The error decrease is faster with optimized coefficients. The solution error is already lower than $1 \%$ with $N=8$. The gain due to the optimization is not lost when adding the first order constraint. The error level is intermediate with the eighth-order constraint in figure $21(\mathrm{~b})$. 


\subsection{Advection of a vortex on overlapping grids}

The first two-dimensional case to study systematically the choice of the interpolation is the advection of a vortex through an overlapping interface [23]. A regular Cartesian background grid of $151 \times 81$ points is overlaid by a smaller foreground grid:

- a $50 \times 51$ regular Cartesian shifted by half a grid spacing as shown in figure $22($ a) with $\Delta x=\Delta y=1 \mathrm{~m}$,

- a $49 \times 49$ sinusoidal grid depicted in figure $22(\mathrm{~b})$, and defined by:

$$
\left\{\begin{array}{l}
x_{i, j}=x_{\min }+(i-1) \Delta x+A \Delta x \sin \left(2 \pi(j-1) \Delta y / L_{y}\right) \\
y_{i, j}=y_{\min }+(j-1) \Delta y+A \Delta y \sin \left(2 \pi(i-1) \Delta x / L_{x}\right)
\end{array}\right.
$$

with $A=1$ and $\Delta x=\Delta y=1 \mathrm{~m}$. The domain size is $L_{x}=x_{\max }-x_{\min } \times$ $L_{y}=y_{\max }-y_{\min }$ with $x_{\min }=30, x_{\max }=70, y_{\min }=-20, y_{\max }=20$.

The initial condition is a Taylor's vortex at the origin $(0,0)$ of the coordinates, defined by:

$$
\left\{\begin{aligned}
u & =u_{\infty}+A \frac{y}{\Delta y} \exp \left(\alpha R^{2}\right) \\
v & =-A \frac{x}{\Delta x} \exp \left(\alpha R^{2}\right) \\
p & =p_{\infty}-\rho_{\infty} \frac{A^{2}}{4 \alpha \Delta x \Delta y} \exp \left(2 \alpha R^{2}\right)
\end{aligned}\right.
$$

where $R=\sqrt{x^{2}-y^{2}}, \alpha=-\ln 2 / b^{2}$ with the half-width $b=0.2 \mathrm{~m}$. The vortex of strength $A=10$ is embedded in a constant mean flow at a Mach number $\mathrm{M}=u_{\infty} / c_{\infty}=0.5$. The computations are run over 800 iterations with $\Delta t=7.22 \times 10^{-4} \mathrm{~s}$. At the last iteration, the vortex is still inside the computational domain but has crossed over the foreground grid. 
Figure 23 shows instantaneous snapshots of the fluctuating pressure when the vortex passes through the first overlapping interface. Second and sixthorder Lagrange interpolations, with stencils of $2 \times 2$ and $6 \times 6$ points respectively, are compared. Intense spurious noise is generated with the secondorder interpolation, whereas the level of noise is less than $\pm 1 \mathrm{~Pa}$ with the sixth-order interpolation. The spurious noise may be quantified by the residual pressure over the first third of the background grid defined as:

$$
R_{p}=\sqrt{\sum_{i=1}^{40} \sum_{j=1}^{81} \frac{\left(p_{i, j}-p_{\infty}\right)^{2}}{40 \times n y_{1}}}
$$

The fall of the residual pressure after the passage of the vortex, plotted in figure 24, helps to quantify the level of spurious noise. For an interpolation on a $6 \times 6$ stencil, the residual pressure is already small. The close up of figure 24(b) shows that the spurious noise is weaker when the order of interpolation increases. The $\mathrm{L}_{2}$-norm error of pressure between the numerical solution and the analytical one at the final iteration is plotted in figure 25 for different interpolation schemes. This error is computed on the last third of the background grid, and is normalized by the error obtained on the background grid alone (i.e. without overlapping).

As for the residual pressure, the error is seen to decrease with the order of interpolation for a Lagrange method. Beyond a $8 \times 8$ interpolation, the error remains very close to the one obtained on the background grid alone. The large errors introduced by a fully optimized interpolation for small stencil are corrected by adding fourth order constraints. For large stencil, the error is not monotonic due to error compensations. Similar results are obtained with the sinusoidal insert in figure 25(b), indicating that the isoparametric mapping 
does not introduce additional errors. The effect of turning off the highorder evaluation of the offsets determining the location of the interpolation point in the transformed space is illustrated in this plot. A second-order approximation of the offsets is then directly provided by the grid-assembly Ogen. A residual error of few percents is then observed and reduce the accuracy for $N>10$. Note that the spectral content for the vortex is in the low wavenumber range, so that the conclusions, similar to that of Sherer and Scott [21], concern well-discretized disturbances.

\subsection{Propagation of an harmonic source on overlapping grids}

The propagation of a Gaussian harmonic source on the same meshes is now considered. This test-case allows the control of the number of grid points per wavelength for a perturbation, by imposing the frequency. The harmonic source, located at the origin, is defined by:

$$
S(t)=\epsilon \sin (\omega t) \exp \left(\frac{\ln 2}{\sigma^{2}}\left(x^{2}+y^{2}\right)\right)
$$

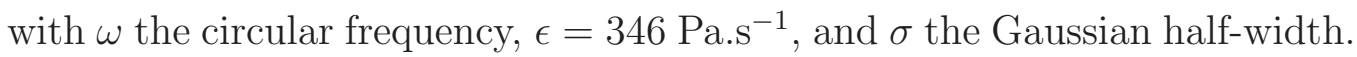
Two values of $\omega$ are tested. The first case is $\omega=2 \pi / 10 \times c_{\infty} / \Delta x, \sigma=3$, which means that the wavelength is equal to 10 mesh spacings. The solution is then well-resolved with respect to the discretization schemes used. Figure 26 presents the $\mathrm{L}_{2}$-norm error between the root mean square numerical and analytical pressures for a cut along $y=0$ with the Lagrange interpolations and the optimized interpolations with or withour order constraints. This error is computed only on the interior grid points of each grids, and is normalized by the error obtained on the background grid alone as previously. Whatever the interpolations used, the errors in figure 26 are comparable beyond a $8 \times 8$ 
stencil and tend toward the error of the computation on the background grid alone. Identical trends are obtained for the foreground sinusoidal grid, indicating that the influence of the mapping is negligible.

The second case is $\omega=2 \pi / 5 \times c_{\infty} / \Delta x$, i.e. 5 points per wavelength. The discretization of the wavelength in this case approaches the limit of resolvability of the spatial finite-difference scheme. The Gaussian half-width is also halved, $\sigma=1.5 \mathrm{~m}$, to obtain a similar compactness of the source.

Figure 27 depicts the same error as figure 26 for the different interpolations. Optimized interpolations improve the solution for a given stencil size. This trend is enhanced for smaller stencils. Moreover, optimized interpolations tend more rapidly toward the reference value on the background grid without overlapping. The disparity of values between a $8 \times 8$ interpolation and a $14 \times 14$ interpolation is less than one per cent of the reference value. Thus, whatever the wavelengh of the source, the introducing of interpolations involves an error which decreases with the size of the stencil. However, even in the more constraining case, the most extented stencil does not present a significant improvement in comparison with the stencils of size $6 \times 6$ or $8 \times 8$. The choice of a stencil smaller than the stencil of the corresponding finitedifference scheme can be acceptable. Moreover, the optimized interpolations with $4^{\text {th }}$-order constraint present globally the better behavior.

\section{Results}

\subsection{Scattering of an harmonic source by two cylinders}

The scattering of an harmonic source by two cylinders is a challenging test case with structured grids. Thanks to overset grid technique, it becomes 
possible to consider three simple grids: two body-fitted polar grids for each cylinder, and a Cartesian background grid to propagate acoustic waves. The first and second cylinder have a radius of $0.5 \mathrm{~m}$ and $0.25 \mathrm{~m}$, and are located at $(x, y)=(-4,0)$ and $(0,4)$ respectively. The resolution of the first polar grid is $n_{r}=25 \times n_{\theta}=181$, and the second polar grid is $n_{r}=29 \times n_{\theta}=181$. The Cartesian grid has a resolution of $n_{x}=164 \times n_{y}=164$. Interpolations are optimized with $4^{\text {th }}$-order constraints on $6 \times 6$ stencils.

The harmonic source defined by (36) has a circular frequency $2 \pi c_{\infty}$, and the half-width of the Gaussian is set to $0.2 \mathrm{~m}$. Figure 28 depicts the instantaneous pressure field. The scattering effect is observed, and is enhanced near the bigger cylinder. The fluctuating pressure in the silence zone behind the cylinders is more intense for the smaller cylinder. No spurious noise allows the detection of the presence of the overlapping interfaces.

Figure 29 presents the root mean square pressure along the line $y=0$ for the numerical solution, compared with the analytical solution given by Sherer [40]. The interference beams are very well reproduced in the region between the two cylinders. Small errors are however visible after the small cylinder, which can not be reduced by increasing the interpolation order. This discrepancy is rather attributed to the non-reflecting boundary conditions, which induce a residual error highlighted in this silence zone.

\subsection{Gust cascade interaction noise}

\subsubsection{Presentation of the problem}

The periodic impingement of the wakes of a rotor on a downstream stator is one of the principal sources of turbomachinery noise, and has been studied extensively using analytical modeling and numerical approaches [41]. 
A simplified configuration has been proposed in the fourth CAA workshop on benchmark problems [42]. The two-dimensional geometry is the unrolled section of a realistic three dimensional fan outlet guide vane stator. Figure 30 shows a sketch of the computational arrangement. The cascade gap-tochord ratio is $S / C=2 / 3$ with inflow and outflow planes located at $\pm 1.5 C$. The fan stage considered has 22 rotor blades and 54 stator vanes. Since both numbers are even, a half stator with 27 vanes is simulated for symmetry reasons. Periodic conditions apply at the upper and lower boundaries. The vane geometry is given in [42].

Euler equations are solved on a set of overlapping grids. A body-fitted O-grid is used around each vane with 201 and 25 points in the directions parallel and normal to the airfoil respectively. The background Cartesian grid is $301 \times 82$ points per passage. The number of points per passage is 29707 (without the hole-cutting process), giving approximately 800000 points for the cascade. The mesh for one passage is illustrated in figure 31. Note that the grid is extended with a geometric stretching after the outflow plane up to $x / C \simeq 4$. This extension combined with a Laplacian dissipation constitutes a sponge zone to damp spurious pressure reflections when the gust is advected. As shown for instance by Nallasamy et al. [43], the reflected waves can interfere with the radiated acoustic waves. One-dimensional characteristic condition [44] are used at the inlet and outlet boundaries to provide the non-reflecting conditions. $2 \times 1-\mathrm{D}$ interpolation optimized with $4^{\text {th }}$-order constraints on a $6 \times 6$ stencil is retained since no high-frequency phenomena are involved. 


\subsubsection{Mean flow results}

The mean flow conditions are set by imposing the outlet pressure $p_{o} / p_{i}=$ 0.92 , and the incoming flow direction $\alpha_{i}=36^{\circ} \cdot p_{i}=1$, and $T_{i}=1$ are the nondimensionalized stagnation pressure and temperature at the inlet. The flow is assumed inviscid and isentropic with reference values $p_{\text {ref }}=$ $101353 \mathrm{~Pa}$, and $T_{\text {ref }}=288 \mathrm{~K}$.

The steady flow is first converged on a single vane passage. The Mach number based on velocity amplitude is 0.449 in the inflow plane $(x / C=-$ $1.5)$ and 0.350 in the outflow plane $(x / C=1.5)$, in good agreement with the reference values 0.449 and 0.347 respectively, provided by Envia [45]. The nondimensionalized pressure at the inlet is 0.872 in the present computation, and 0.870 with the code Turbo in [45]. The steady loading on the vane is plotted in figure 32. A very good agreement is obtained with Turbo reference [45], and with the results of Nallasamy et al. [43]. Only small discrepancies are visible at the trailing edge, which is sensitive to the particular treatment of this edge point. The close up of pressure contours in figure 33 indicates that no disturbances are generated by the overset interfaces.

\subsubsection{Gust definition}

The inflow periodic wake disturbance is modeled by a gust imposed at the inlet boundary as:

$$
\begin{aligned}
u_{g}^{\prime}(y, t) & =a_{1} \cos \left(k_{y} y-\omega t\right)+a_{2} \cos \left(2\left(k_{y} y-\omega t\right)\right) \\
& +a_{3} \cos \left(3\left(k_{y} y-\omega t\right)\right) \mathbf{e}_{\beta} \\
\rho_{g}^{\prime}(y, t) & =0, \quad p_{g}^{\prime}(y, t)=0
\end{aligned}
$$


where the angle $\beta=44^{\circ}$ defines the direction of the gust $\mathbf{e}_{\beta}=\cos \beta \mathbf{e}_{x}-$ $\sin \beta \mathbf{e}_{y}$. The fundamental reduced frequency $\omega$ is fixed at $3 \pi / 4$, and the transverse wavenumber is $k_{y}=11 \pi / 9$. The gust is the sum of the first three harmonics of the blade passing frequency (BPF) $\omega$. Their amplitudes are $a_{1}=5 \times 10^{-3}, a_{2}=3 \times 10^{-3}$, and $a_{3}=7 \times 10^{-4}$. As verified by Nallasamy et al. [43], the response to these gust amplitudes remains linear. That is why several authors have used linearized solvers $[45,46]$. In the present study, the calculations are performed separately for single frequency excitations at $\mathrm{BPF}, 2 \mathrm{BPF}$ and $3 \mathrm{BPF}$.

\subsubsection{Tyler-Sofrin modes}

The rotor-stator interaction generates acoustic tones satifying the TylerSofrin criterion. Tyler and Sofrin [47] have studied spinning pressure patterns in compressors. Each harmonic frequency may have one or several circumferencial modes that propagate or decay. If $N_{\text {rotor }}$ is the number of blades of the rotor, and $N_{\text {stator }}$ the number of vanes of the stator, the order of the circumferencial modes satisfy:

$$
m=n N_{\text {rotor }}+k N_{\text {stator }}
$$

where $n$ is the BPF harmonic number, and $k$ is an integer. In simple terms, an acoustic pulse is generated when a blade and a vane are coincident. Consider the visual interpretation in figure 34 adapted from Tyler and Sofrin [47]. In the first row, the rotor has 3 blades and the stator 4 vanes. Using $k=-1$ and the first harmonic in (38) yields $m=3-4=-1$, meaning that the direction of rotation of the generated pressure waves is opposite to that of the rotor. In the second example (second row in figure 34), the 4 blades of 
the rotor generate acoustic pulses when they are passing in front of one of the 12 vanes of the stator. Formula (38) for the first harmonic and $k=0$ gives $m=4-0=+4$. The spinning mode has four lobes (order 4 ), and rotates in the same direction as the rotor ( + sign).

The fan duct then acts as a filter. Depending on its wavenumber, the mode can propagate (cut-on mode), or is evanescent (cut-off mode). In the present configuration, the fan stage is composed of 22 rotor blades and 54 stator vanes. At the first harmonic $(n=1)$, the lowest order modes are $m=22$ $(k=0)$, and $m=-32(k=1)$. Nallasamy et al. [43] have calculated their cut-off ratios, and show that both modes are evanescent. At the second harmonic, the dominant mode is obtained for $k=1$, yielding a counter-spinning mode $m=-10$, which is shown to be propagative. Two modes of order $m=12(k=1)$, and $m=-42(k=2)$ are cut-on at the third BPF harmonic.

\subsubsection{Acoustic results on the overlapping grids}

The gust (37) is imposed through a triple decomposition $u=\bar{u}+u_{g}+u^{\prime}$ in the inlet characteristic condition. The results for the first harmonic $n=1$ with amplitude $a_{1}$ are depicted in figure 35. The advection of the velocity gust is illustrated in plots (a) and (b), and represents the impingement of the rotor blade wakes on the stator vanes. In particular, no oscillations are noticed after the inlet demonstrating the effectiveness of the triple decomposition for the inlet condition. The clean imposition of the gust is also effective for the two other frequencies (not shown for brievety). The pressure patterns in figure 35(c) are in excellent agreement with previous results [45, 48, 43]. The mode is cut-off, and an exponentional decay of the pressure upstream and downstream of the airfoil is observed. The unsteady pressure distribu- 
tion over a vane is compared quantitatively with the results from Coupland [48], Nallasamy et al. [43], and Wang et al. [49] in figure 36. A very good agreement is observed with Coupland's simulation performed on a very fine mesh. The distribution has the same shape for the two other references, but Nallasamy et al. predict higher levels $(+2.5 \mathrm{~dB})$ and Wang et al. underpredict the levels $(-4 \mathrm{~dB})$. These trends are conform with the overall pressure levels in the histograms of figure 39.

The propagating acoustic modes for the second and third harmonic are illustrated in figure 37. The mode $m=-10$ for $n=2 \mathrm{BPF}$ is spinning in the direction opposite of the rotor. Five wavelengthes are visible upstream and downstream, corresponding to a circumferencial order $\left|m^{\prime}\right|=5$ (the half of -10 since only a half stator is calculated). Two modes are distinguishable in figure $37(\mathrm{~b})$ for $n=3 \mathrm{BPF}$. Between the inflow and the vane, a mode with a weak intensity has 6 transverse wavelengthes for the 27 vane cascade, thus $\left|m^{\prime}\right|=6$. This the co-spinning mode $m=12$. After the vane, a mode with 21 wavelengthes is dominant, corresponding to the counter-spinning mode $m=-$ 42. Nallasamy et al. [43] have noted that the mode -42 has a cut-off ratio close to one. This can be related to the particular shape of the mode with almost horizontal lobes. These pressure patterns are in good agreement with those of the benchmark participants.

A quantitative comparison is provided by the unsteady pressure distribution at the highest frequency $n=3$ in figure 38 . The same adjustment has been done for the levels as in figure 36. The global shape agreement is correct even if more scattering is noticed in the details of the distributions. The pressure levels are approximately ten times lower than those at the fundamental 
frequency, so that subtle interferences are more tricky to capture. Note that the discrepancies for the mean level of fluctuations are exactly the same as those noted at the fundamental, indicating that they are not related to the acoustic generation process, but rather to a slight difference in the intensity of the incoming gust. These differences can result from the treatment of the inlet conditions.

Finally, the integrated pressure levels on the vane pressure and suction sides, and at the outflow plane are compared to the values reported in the benchmark in figure 39. Only 3 of the 12 locations asked in the case definition are reported for brievety, but similar hierarchy is observed at the other points. Few decibel differences are noticed as discussed previously, but in general the present overlapping strategy provides satisfactorily results for this challenging benchmark case. In terms of efficiency, the grid used around the vane cannot be reduced in the axial direction due to the size of the overlapping region with an eleven-point stencil scheme. Nevertheless, this constraint will be quickly relaxed when viscous three-dimensional cases are tackled, since a finer resolution is required to capture the boundary layer over the airfoil. Moreover, the interpolation procedure can also be used to define a sliding interface, as in [35] in order to take into account the rotor, and have a more realistic description of the incoming wakes.

\section{Conclusions}

The accuracy of interpolation schemes when used in conjunction with high-order numerical algorithms has been investigated. Explicit Lagrange or optimized interpolations are studied. The choice of the stencil size and of 
the way to compute the coefficients are guided by the precision of the discretization schemes, and by the necessity or not to resolve high-wavenumber perturbations on a given grid. In agreement with the Fourier analysis, the a posteriori error analysis indicates that a DRP-type optimization is advantageous to deal with high-frequency waves. On the other side, Lagrange interpolations maximizing the order of Taylor truncation show lower errors for well-resolved waves. An interesting trade-off is obtained by adding order constraint to the optimization, which can be useful for broadband noise or developed turbulence, where a large range of scales is involved. The analysis also underlined that using an interpolation stencil with less points than a corresponding finite-difference discretization can provide acceptable results. This point is attractive to reduce the cost due to interpolations, which becomes important for more than one space dimension.

The choice of an interpolation for multidimensional curvilinear grids is discussed. The computation of the coefficient directly in the physical space can yield ill-conditioned linear system, so that this option is hardly practicable with large stencils. A mapping of the coordinates of the interpolated point in the computational space allows the tensorization of one-dimensional interpolations by direction. A high-order extension of the isoparametric mapping is retained. It is based on Newton's iterations and avoids the inversion of matrices. The a posteriori tests shows that the error due to the mapping phase is negligible, so that conclusions drawn in 1-D are still valid in 2-D.

Two aeroacoustic benchmarks are conducted in the result part to show that the accuracy of the numerical algorithms is preserved with overlapping grids. The grids for the diffraction from two cylinders are straightforward to 
design by combining polar and Cartesian subdomains, and yield satisfactorily results. The calculation of Tyler-Sofrin modes in the rotor-stator is a more challenging case. Both propagating and evanescent waves are captured, and the interpolation procedure is not the leading parameter for the solution quality. The benefit of the present spectral-like schemes will be more evident for three-dimensional turbulent configurations. When large-eddy simulation is used for instance, the full range of wavenumbers until the scheme cut-off are present, and the grids are fine near the solid boundaries so that it is easier to define the overlapping region with large stencil. Optimized interpolation with fourth-order constraint, and a stencil with two points less than the finite-difference stencil can then provide a good trade-off between efficiency and accuracy. The recent calculation of noise from a turbulent boundary layer over a cylindrical cavity [50] gives an illustration of the interest of the overset strategy in turbulent conditions.

\section{Acknowledgments}

This work was granted access to the HPC resources of IDRIS under the allocation 2010-1736 made by GENCI (Grand Equipement National de Calcul Intensif). The authors gratefully acknowledge funding by FRAE (Fondation de Recherche pour l'Aéronautique et l'Espace) in the AEROCAV project of the IROQUA network.

\section{References}

[1] J. Freund, S. Lele, P. Moin, Numerical simulation of a Mach 1.92 turbulent jet and its sound field, AIAA Journal 38 (11) (2000) 2023-2031. 
[2] C. Bogey, C. Bailly, An analysis of the correlations between the turbulent flow and the sound pressure field of subsonic jets, J. Fluid Mech. 583 (2007) 71-97.

[3] C. Rowley, T. Colonius, A. Basu, On self-sustained oscillations in twodimensional compressible flow over rectangular cavities, J. Fluid Mech. 455 (2002) 315-346.

[4] X. Gloerfelt, C. Bogey, C. Bailly, Numerical evidence of mode switching in the flow-induced oscillations by a cavity, Int. J. of Aeroacoustics 2 (2) (2003) 99-124.

[5] C. Tam, Computational aeroacoustics: issues and methods, AIAA Journal 33 (10) (1995) 1788-1796.

[6] T. Colonius, S. Lele, Computational aeroacoustics : progress on nonlinear problems of sound generation, Progress in Aerospace Sciences 40 (2004) 345-416.

[7] M. Visbal, D. Rizzetta, Large-Eddy Simulation on curvilinear grids using compact differencing and filtering schemes, ASME Journal of Fluids Engineering 124 (2002) 836-847.

[8] O. Marsden, C. Bogey, C. Bailly, Direct noise computation around a turbulent flow around a zero-incidence airfoil, AIAA Journal 46 (4) (2008) $874-883$.

[9] J. Benek, J. Steger, F. Dougherty, A flexible grid embedding technique with application to the Euler equations, AIAA Paper 83-1944. 
[10] B. Kreiss, Construction of a curvilinear grid, SIAM J. Sci. Stat. Comput. 4 (2) (1983) 270-279.

[11] G. Chessire, W. Henshaw, Composite overlapping meshes for the solution of partial differential equations, J. Comput. Phys. 90 (1990) 1-64.

[12] N. Petersson, An algorithm for assembling overlapping grid systems, SIAM J. Sci. Comput. 20 (6) (1999) 1995-2022.

[13] B. Henshaw, K. Chand, D. Quinlan, OvertureHttps://computation.llnl.gov/casc/Overture/.

[14] W. D. Henshaw, D. W. Schwendeman, Parallel computation of threedimensional flows using overlapping grids with adaptive mesh refinement, J. Comput. Phys. 227 (2008) 7469-7502.

[15] O. Saunier, C. Benoit, G. Jeanfaivre, A. Lerat, Third-order Cartesian overset mesh adaptation method for solving steady compressible flows, Int. J. Numer. Meth. Fluids 57 (2008) 811-838.

[16] J. Sitaraman, M. Floros, A. Wissink, M. Potsdam, Parallel domain connectivity algorithm for unsteady flow computations using overlapping and adaptive grids, J. Comput. Phys. 229 (2010) 4703-4723.

[17] S. Péron, C. Benoit, Off-body overset adaptive Cartesian mesh method based on an octree approach, AIAA Paper 2011-3050.

[18] N. Prewitt, D. Belk, W. Shyy, Parallel computing of overset grids for aerodynamic problems with moving objects, Progress in Aerospace Sciences 36 (2000) 117-172. 
[19] J. Yin, J. Delfs, Sound generation from gust-airfoil interaction using CAA-Chimera method, AIAA Paper 2001-2136.

[20] C. Tam, J. Webb, Dispersion-relation-preserving finite difference schemes for computational acoustics, J. Comput. Phys. 107 (1993) 262281.

[21] S. Sherer, J. Scott, High-order compact finite-difference methods on general overset grids, J. Comput. Phys. 210 (2005) 459-496.

[22] G. Desquesnes, M. Terracol, E. Manoha, P. Sagaut, On the use of a high order overlapping grid method for coupling in CFD/CAA, J. Comput. Phys. 220 (2006) 355-382.

[23] T. Emmert, Development of a multidomain high-order algorithm for computational aeroacoustics: application to subsonic and transonic confined flows, Ph.D. thesis, Ecole Centrale de Lyon, no 2007-30 (2007).

[24] F. Daude, J. Berland, T. Emmert, P. Lafon, F. Crouzet, C. Bailly, A high-order finite-difference algorithm for direct computation of aerodynamic sound, Computers and Fluids 61 (2012) 46-63.

[25] D. Desvigne, O. Marsden, C. Bogey, C. Bailly, Development of noncentered wavenumber-based optimized interpolation schemes with amplification control for overlapping grids, SIAM J. Sci. Comput. 32 (4) (2010) 2074-2098.

[26] C. Tam, K. Kurbatskii, A wavenumber based extrapolation and interpolation method for use in conjunction with high-order finite difference schemes, J. Comput. Phys. 157 (2000) 588-617. 
[27] M. Berger, On conservation at grid interfaces, SIAM J. Num. Analysis 24 (1987) 967-983.

[28] G. Chessire, W. Henshaw, A scheme for conservative interpolation on overlapping grids, J. Comput. Phys. 15 (1994) 819-845.

[29] Z. Wang, A fully conservative interface algorithm for overlapped grids, J. Comput. Phys. 122 (1995) 96-106.

[30] T. Emmert, P. Lafon, C. Bailly, Computation of aeroacoustic phenomena in subsonic and transonic ducted flow, 13th AIAA/CEAS AeroAcoustics Conference, 21-23 May, Rome, Italy, AIAA Paper 2007-3429.

[31] J. Banks, D. Schwendeman, A. Kapila, W. Henshaw, A high-resolution Godunov method for compressible multi-material flow on overlapping grids, J. Comput. Phys. 223 (2007) 262297.

[32] J. Delfs, An overlapped grid technique for high resolution CAA schemes for complex geometries, AIAA Paper 2001-2199.

[33] R. Guénanff, Couplage instationnaire Navier-Stokes/Euler pour la génération et le rayonnement des sources de bruit aérodynamique, Ph.D. thesis, Université Rennes I, no 3138 (2004).

[34] O. Marsden, C. Bogey, C. Bailly, High-order curvilinear simulations of flows around non-Cartesian bodies, J. Comput. Acous. 13 (4) (2005) $731-748$.

[35] C. Tam, F. Hu, An optimized multi-dimensional interpolation scheme 
for computational aeroacoustics applications using overset grids, AIAA Paper 2004-2812.

[36] R. Guénanff, M. Terracol, E. Manoha, P. Sagaut, R. Lewandowski, Theoretical and numerical aspects of a multidomain high-order method for CAA, AIAA Paper 2003-3117.

[37] T. Le Garrec, X. Gloerfelt, C. Corre, Multi-size-mesh, multi-time-step algorithm for noise computation around an airfoil in curvilinear meshes, 13th AIAA/CEAS AeroAcoustics Conference, 21-23 May, Rome, Italy, AIAA Paper 2007-3504.

[38] C. Bogey, C. Bailly, A family of low dispersive and low dissipative explicit schemes for noise computation, J. Comput. Phys. 194 (2004) 194214.

[39] T. Le Garrec, Simulation direct du bruit de bord de fuite d'un profil par une méthode multi domaines, Ph.D. thesis, ENSAM, no ENAM 0021 (2008).

[40] S. Sherer, Scattering of sound from axisymmetric source by multiple circular cylinders, J. Acoust. Soc. Am. 115 (2) (2004) 488-496.

[41] E. Envia, A. Wilson, D. Huff, Fan noise: a challenge to CAA, International Journal of Computational Fluid Dynamics 18 (6) (2004) 471-480.

[42] E. Envia, Category 3, Sound generation by interacting with a gust, Problem 2, Cascade-gust interaction, Fourth Computational Aeroacoustics (CAA) Workshop on Benchmark Problems, NASA CP 2004-212954 (2004) 18-22. 
[43] M. Nallasamy, R. Hixon, S. Sawyer, Solution of unsteady Euler equations: Gust-cascade interaction tones, Computers and Fluids 36 (2007) $724-741$.

[44] K. Thompson, Time dependent boundary conditions for hyperbolic systems, J. Comput. Phys. 68 (1987) 1-24.

[45] E. Envia, Benchmark solution for the Category 3-Problem 2: Cascadegust interaction, Fourth Computational Aeroacoustics (CAA) Workshop on Benchmark Problems, NASA CP 2004-212954 (2004) 59-63.

[46] A. Escribano, A. Serrano, C. Vasco, Cascade-gust-interaction problem analysis based on linear CFD calculations, Fourth Computational Aeroacoustics (CAA) Workshop on Benchmark Problems, NASA CP 2004-212954 (2004) 81-88.

[47] L. Tyler, T. Sofrin, Axial flow compressor noise studies, Trans. SAE 70 (1962) 309-332.

[48] J. Coupland, Linear unsteady CFD analysis of the cascade-gust interaction problem, Fourth Computational Aeroacoustics (CAA) Workshop on Benchmark Problems, NASA CP 2004-212954 (2004) 97-105.

[49] X.-Y. Wang, A. Himansu, S.-C. Chang, P. Jorgenson, Computation of a single airfoil gust response and gust-cascade interaction using the CE/SE method, Fourth Computational Aeroacoustics (CAA) Workshop on Benchmark Problems, NASA CP 2004-212954 (2004) 115-126.

[50] J. Chicheportiche, X. Gloerfelt, Effect of a turbulent incoming boundary layer radiation by the flow over cylindrical cavities, 17th AIAA/CEAS 
AeroAcoustics Conference, 6-8 June, Portland, Oregon, AIAA Paper 2011-2588.

[51] R. Hixon, Solution of category 3, problem 2, using the Space-Time Mapping Analysis (STMA) method, Fourth Computational Aeroacoustics (CAA) Workshop on Benchmark Problems, NASA CP 2004-212954 (2004) 127-134.

[52] M. Nallasamy, R. Hixon, S. Sawyer, R. Dyson, Category 3, Sound generation by interacting with a gust, Problem 2, Cascade-gust interaction, Fourth Computational Aeroacoustics (CAA) Workshop on Benchmark Problems, NASA CP 2004-212954 (2004) 89-96. 


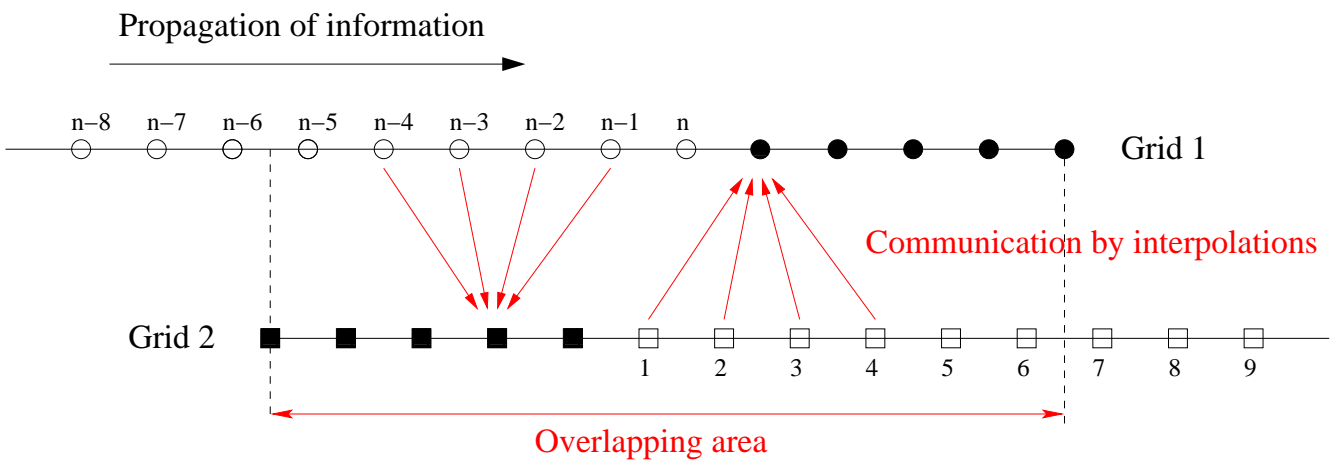

Figure 1: 1-D example of two overlapping grids. Each grid is both receiver and donor of information. The black points are the interpolation points, called ghost points. The white points are interior points on which derivatives are computed on a centered eleven-point stencil. Thus, five ghost points in the overlapping area are needed. The four arrows symbolize an explicit interpolation from a 4-point stencil. 


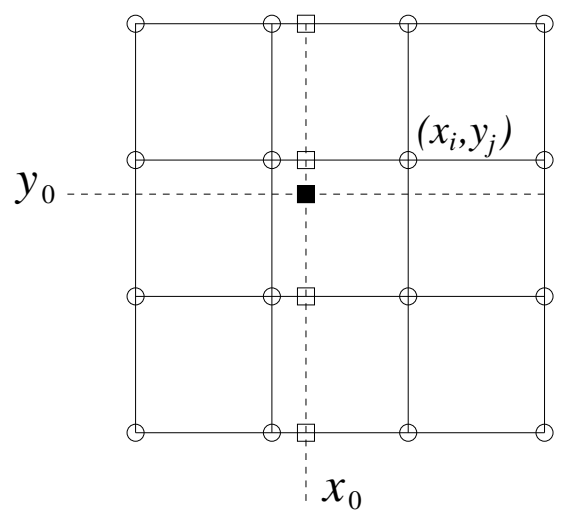

Figure 2: 2-D Cartesian interpolation stencil. White circles denote the points of the interpolation stencil, the black square is the desired interpolated point, and white squares are the intermediate interpolated values. 


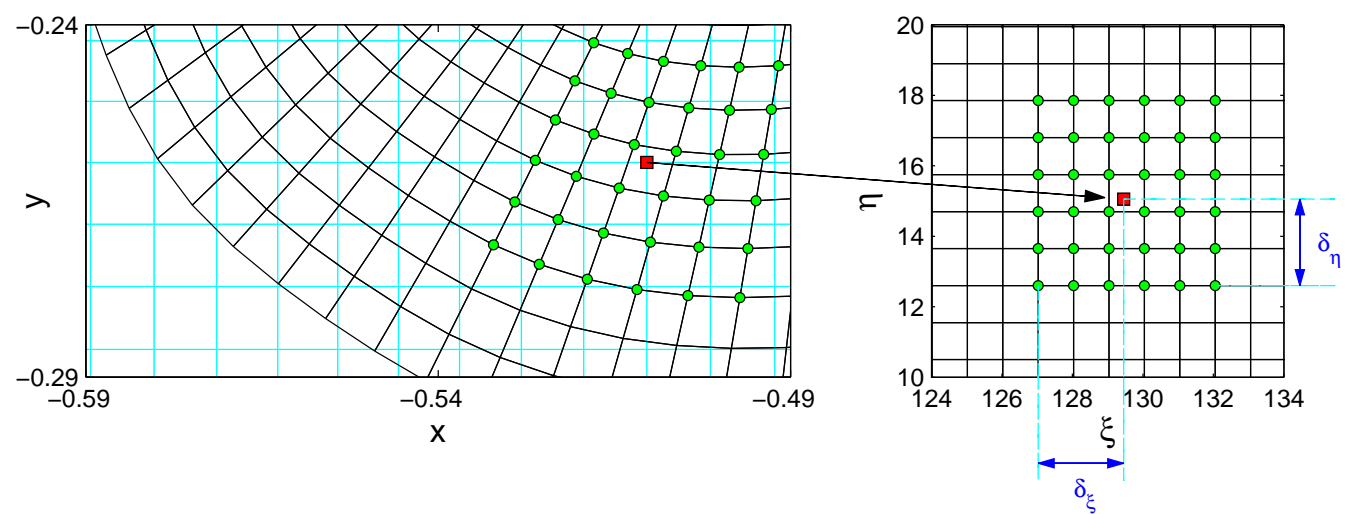

Figure 3: Sketch of the mapping from the physical space $(x, y)$ to the computational space $(\xi, \eta)$. The example on the left is a zoom of the overlapping grids used later in $\S 6.2$ : the Cartesian grid is the background grid (— ) which contains the interpolation point (red square). The curvilinear grid (- ) is an O-grid around the vane (the zoom represents the lower upstream part). Donor points (green circles) belong to the curvilinear grid. The right insert show the mapping of the curvilinear into a Cartesian regular grid with unit spacing. The interpolation point is located in this space by the offsets $\left(\delta_{\xi}, \delta_{\eta}\right)$ relative to the base point of the stencil (lower left donor point). 

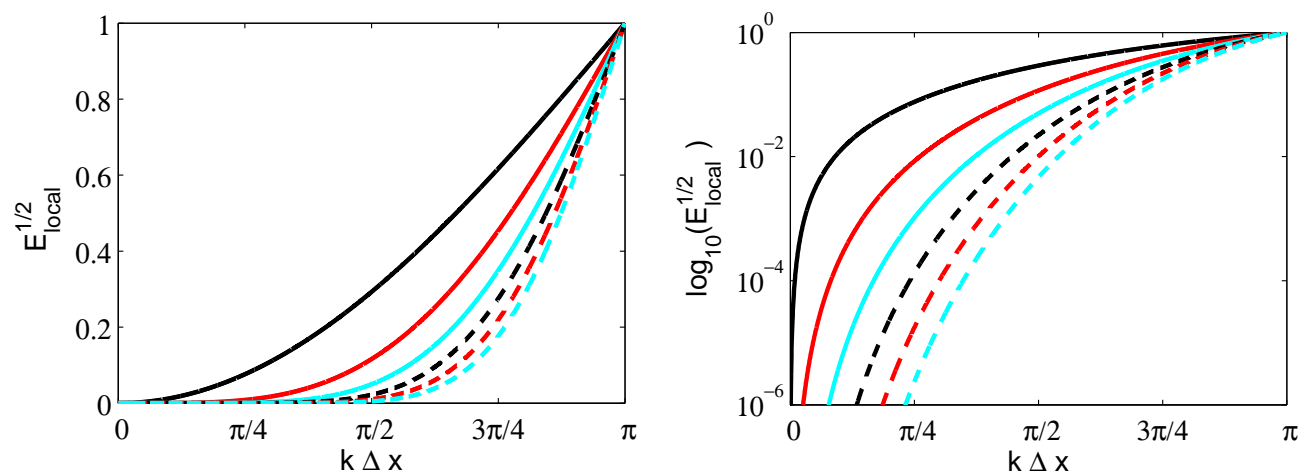

Figure 4: Local error in the wavenumber space for the Lagrange interpolation with stencil of size $N=2(-), N=4(-), N=6(-), N=8(---), N=10(-$ - - ), and $N=12(---)$. Linear scale on top, and logatirhmic scale on bottom. 

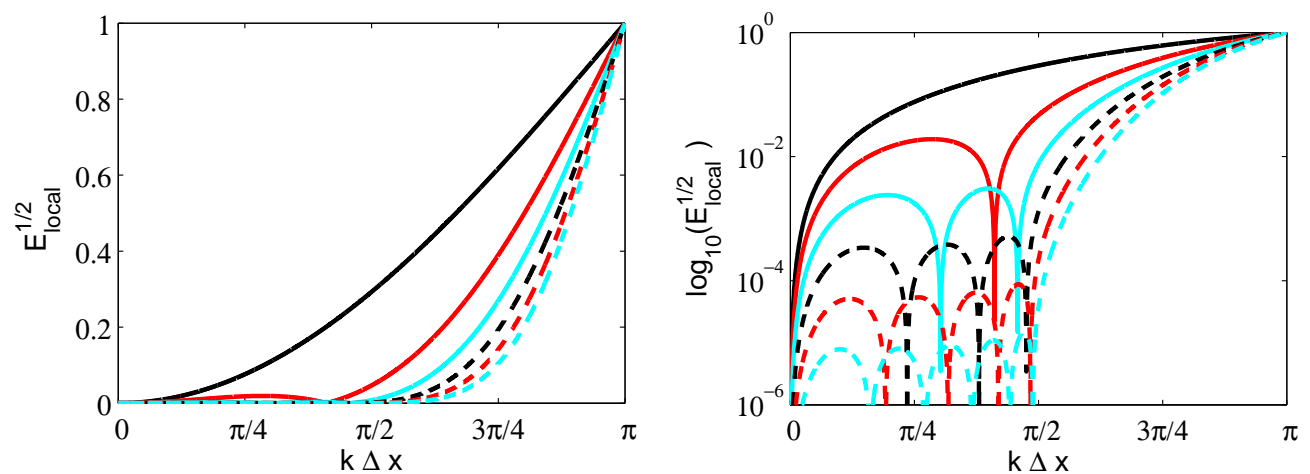

Figure 5: Local error in the wavenumber space for the optimized interpolation with stencil of size $N=2(-), N=4(-), N=6(-), N=8(---), N=10(-$ $--)$, and $N=12(---)$. 

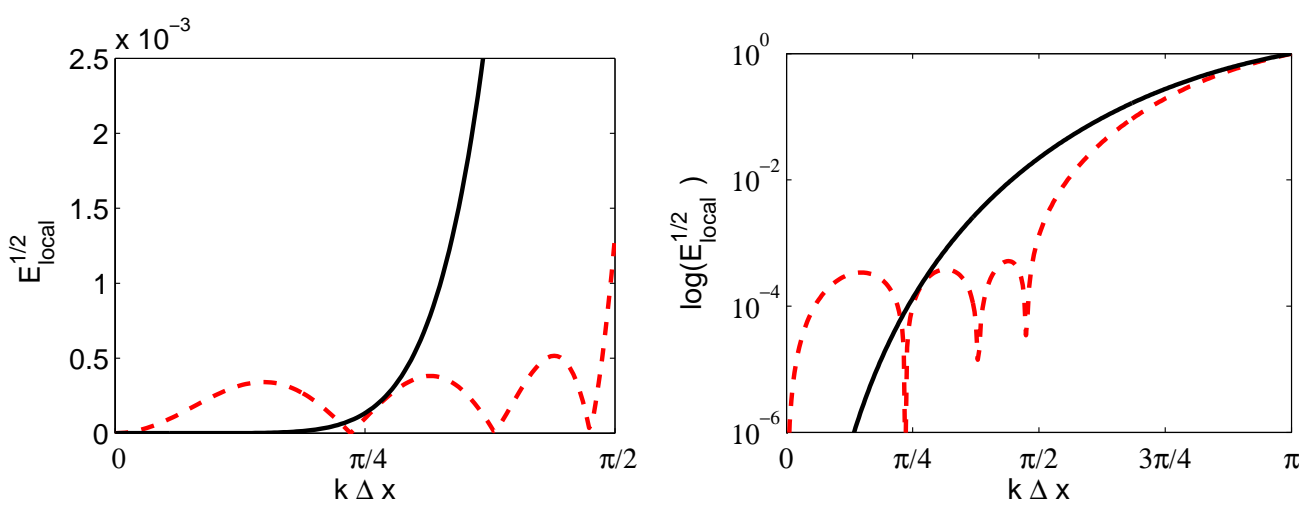

Figure 6: Comparison of the local error in the wavenumber space, obtained with Lagrange interpolation ( - ) and optimized interpolation (- - - ), for $N=8$. 

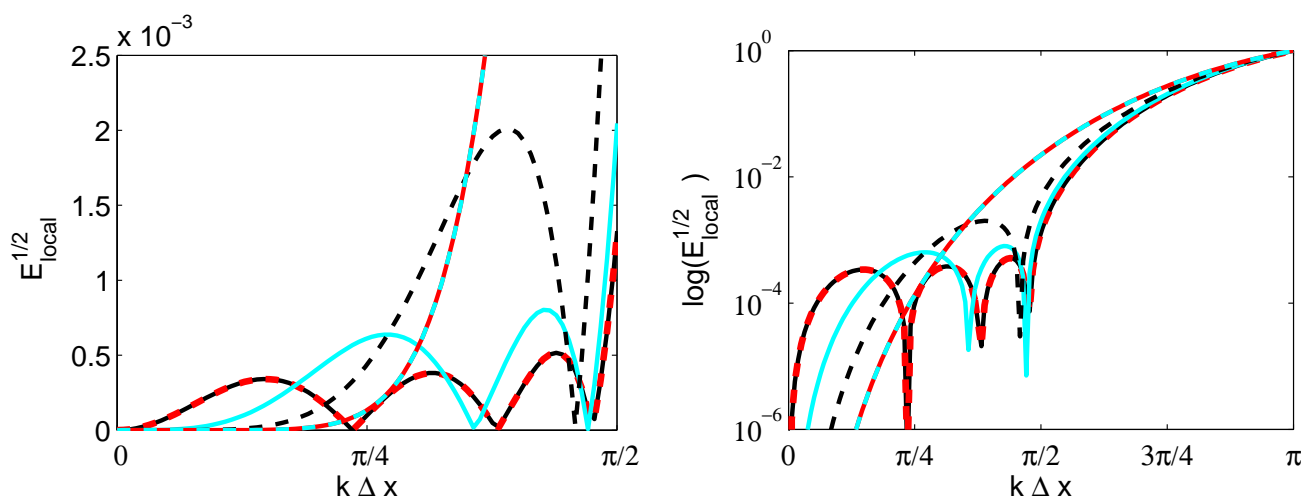

Figure 7: Local error in the wavenumber space for the optimized interpolation with order constraints for a stencil size $N=8$. Interpolation of order (о.) : o.0 ( - ), o.2 ( - - $)$, o.4 ( $(-)$, o.6 ( - - $)$, o.8 ( - - $)$. Lagrange interpolation for $N=8(-)$. 


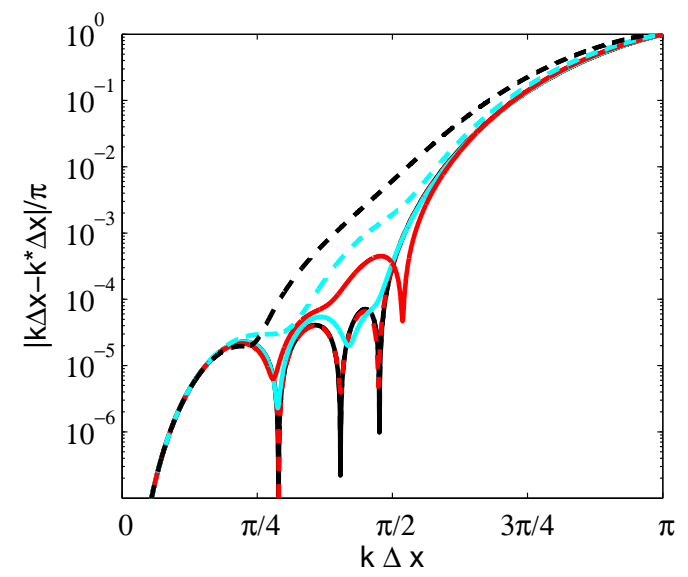

Figure 8: Dispersion error of a scheme combining an eleven-point derivative and $q$ interpolations: $q=1(---), q=2(-), q=3(-), q=4(---)$, $q=5(---)$. Dispersion error of the finite-difference derivative alone $(-)$ is plotted as a reference. Lagrange interpolations are used with a stencil of size $N=8$. 


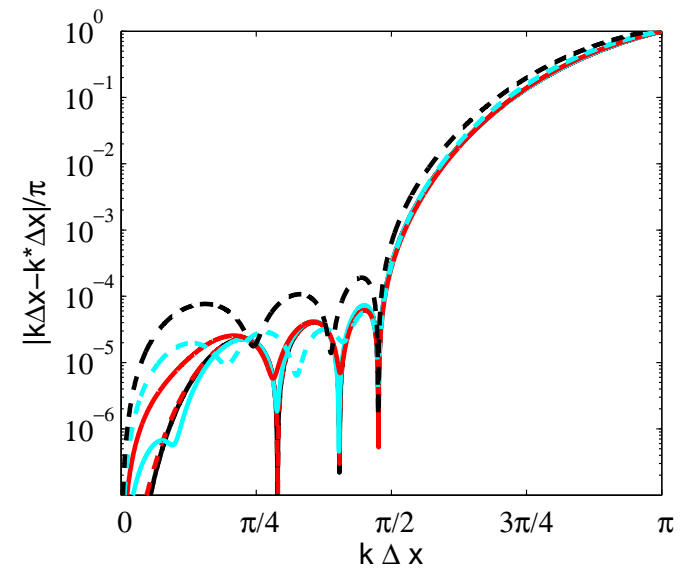

Figure 9: Same legend as figure 8 for fully optimized interpolations on a $N=8$ stencil. 


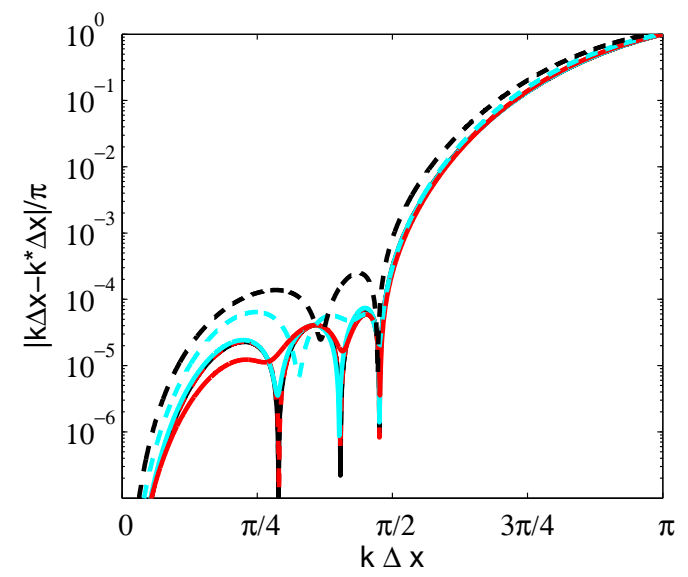

Figure 10: Same legend as figure 8 for optimized interpolations with fourth-order constraint on a $N=8$ stencil. 




Figure 11: Same legend as figure 8 for optimized interpolations with sixth-order constraint on a $N=8$ stencil. 


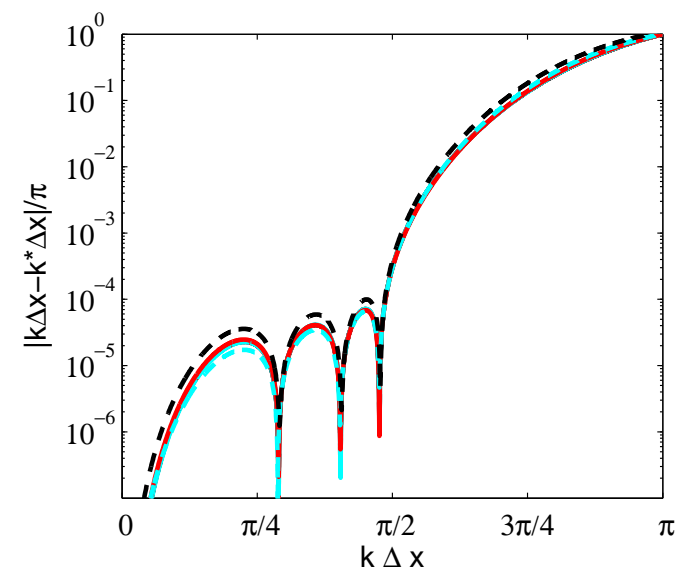

Figure 12: Same legend as figure 8 for optimized interpolations with fourth-order constraint on a $N=10$ stencil. 


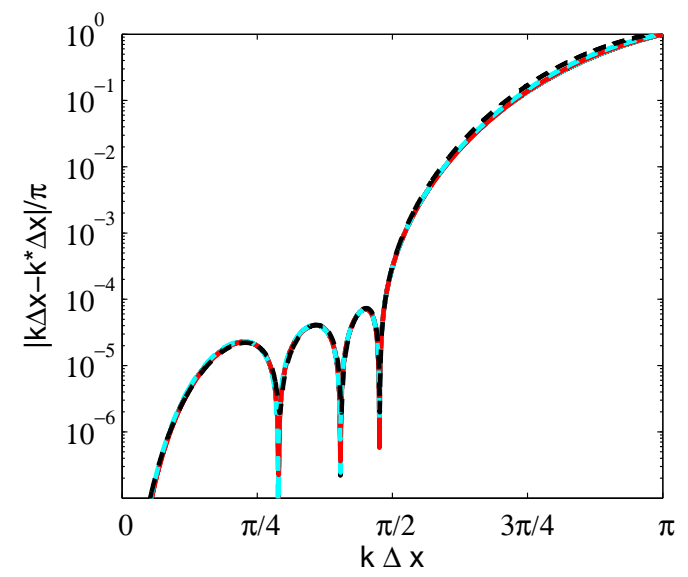

Figure 13: Same legend as figure 8 for optimized interpolations with fourth-order constraint on a $N=12$ stencil. 


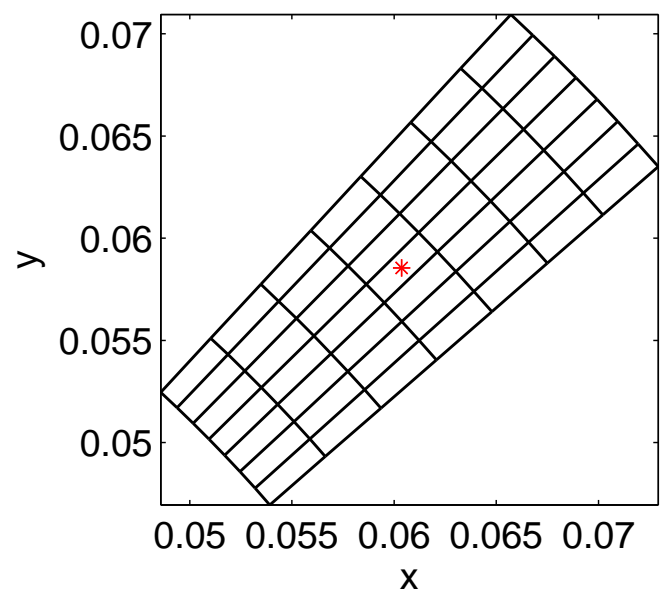

Figure 14: Curvilinear $8 \times 8$ stencil used for the spectral analysis of 2-D interpolation methods; $\Delta r=0.0036$, and $\Delta \theta=2 \pi / 410$. 


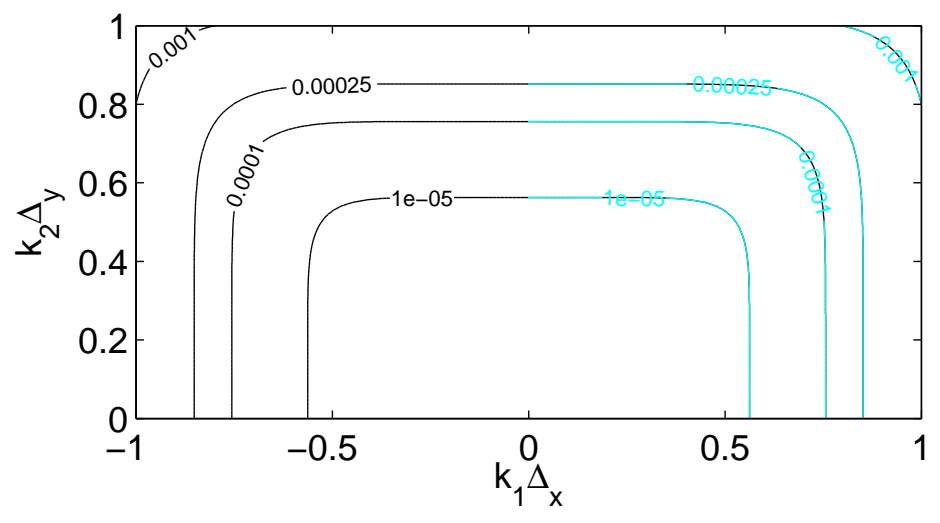

(a)

(b)
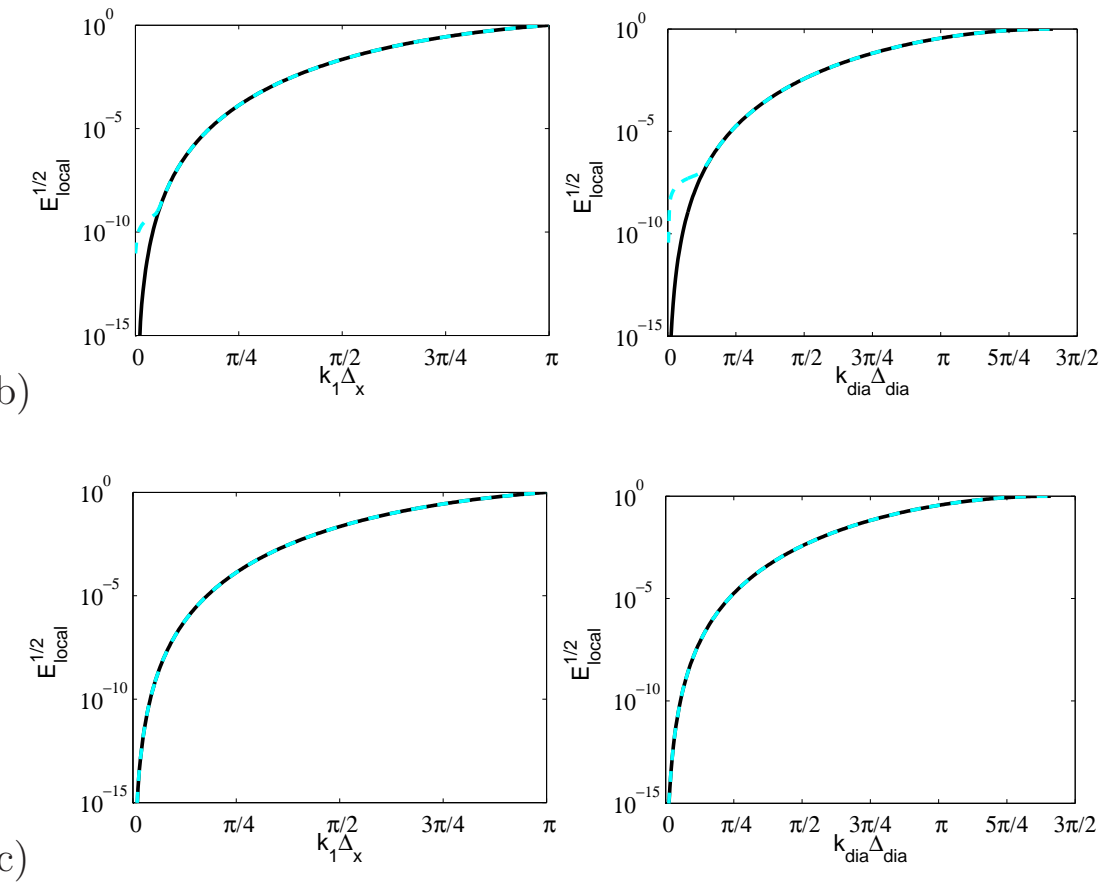

Figure 15: Effect of the choice of the mapping for $2 \times 1-\mathrm{D}$ interpolations. (a) Map of the two-dimensional local error for an interpolation using a polynomial approximation of the inverse mapping (— $)$, superimposed on the reference error using the polar mapping (-). (b) Profiles of the error along $k_{1} \Delta_{x}$ (left) and along $k_{1} \Delta_{x}=k_{2} \Delta_{y}$ (right) for polynomial inverse mapping ( - - - ). (c) Same profiles for the isoparametric mapping $(---)$ compared to the reference case with polar mapping $(-)$. 


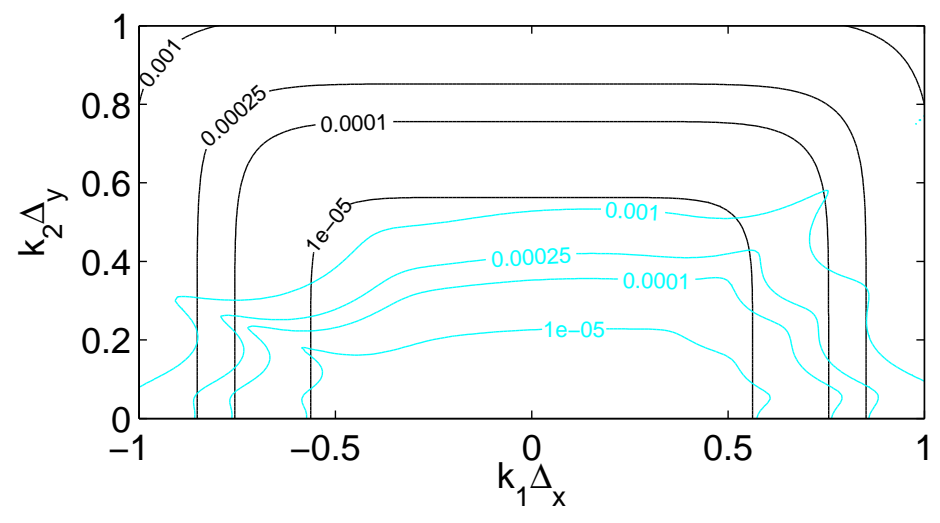

(a)


(b)
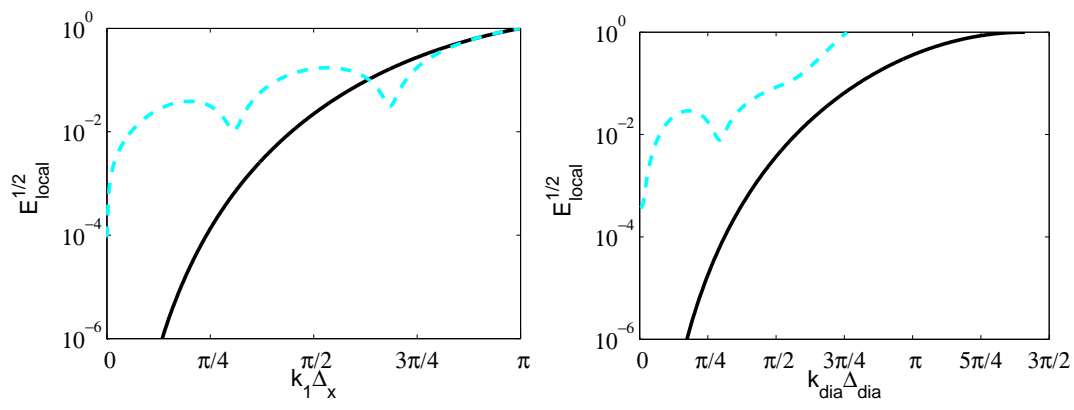

Figure 16: Interpolation in the curvilinear space. (a) Map of the two-dimensional local error for a multidimensional interpolation ( - ), superimposed on the reference error using the polar mapping (- ). (b) Profiles of the error along $k_{1} \Delta_{x}$ (left) and along $k_{1} \Delta_{x}=k_{2} \Delta_{y}$ (right) for the standard multidimensional interpolation $(---)$. (c) Same profiles for the optimized multidimensional interpolation $(---)$. 


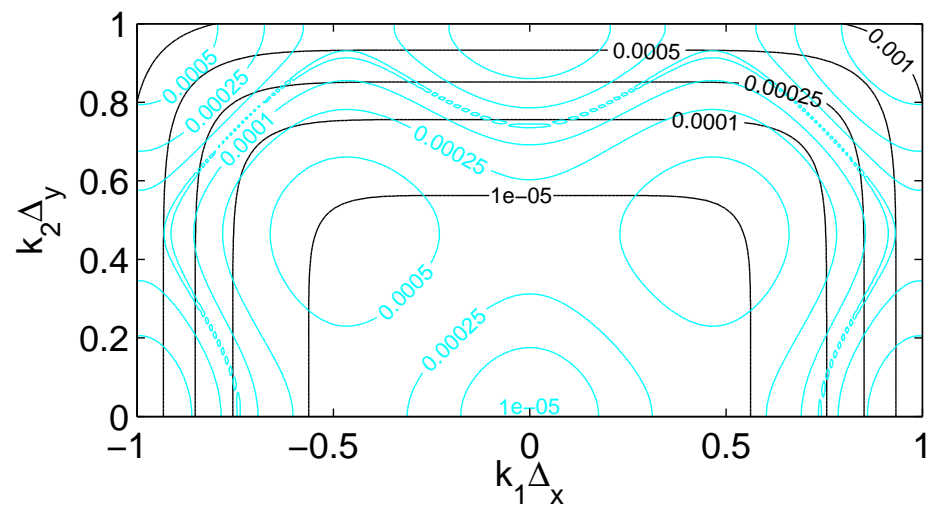

(a)

(b)
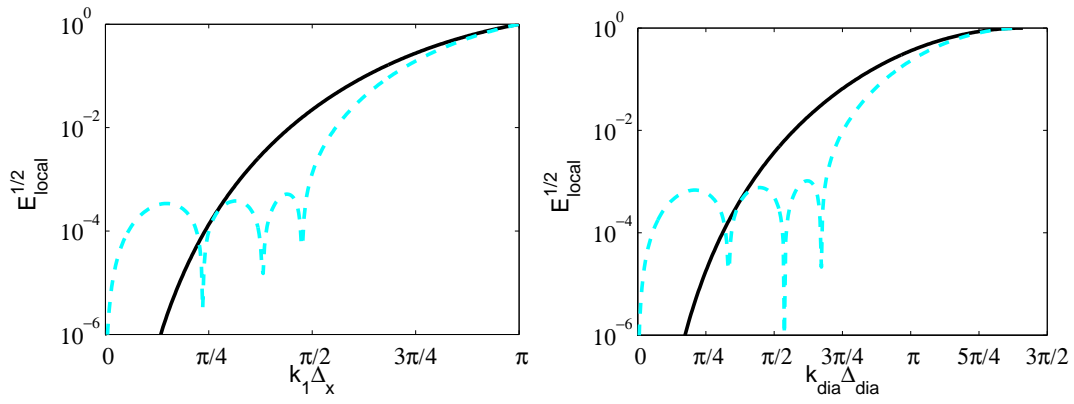

(c)
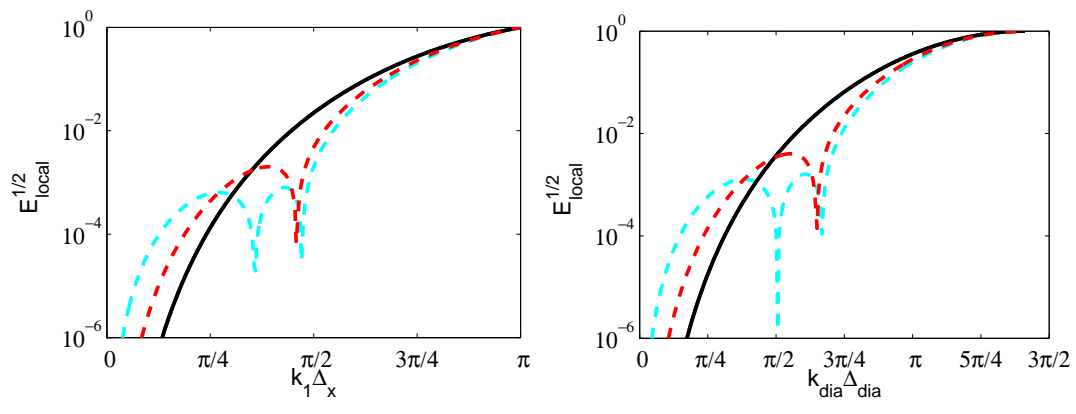

Figure 17: Optimized $2 \times 1 \mathrm{D}$ interpolation with or without order constraints. (a) Map of the two-dimensional local error for an optimized $2 \times 1$-D interpolation (), superimposed on the reference error using the polar mapping (- ). (b) Profiles of the error along $k_{1} \Delta_{x}$ (left) and along $k_{1} \Delta_{x}=k_{2} \Delta_{y}$ (right) for the fully optimized $2 \times 1$-D interpolation $(---$ ). (c) Same profiles for the optimized $2 \times 1-\mathrm{D}$ interpolation with fourth-order $(---)$ or sixth-order $(---)$ constraint. 

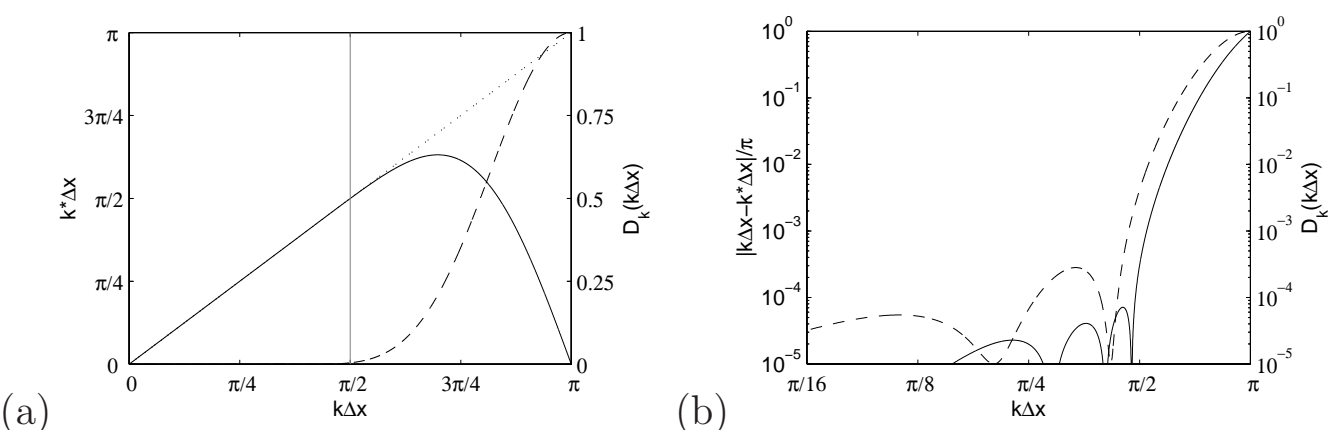

(b)

Figure 18: Properties of schemes in the wavenumber space $k \Delta x$. (a) The effective wavenumber $k^{*} \Delta x$ of the eleven-point-stencil optimized scheme ( - ), and the damping function $D_{k}(k \Delta x)$ of the eleven-point stencil optimized filter ( - - ). (b) The dissipation errors $\left|k \Delta x-k^{*} \Delta x\right| / \pi$ for the discretization schemes and $D_{k}(k \Delta x)$ for the optimized filter are shown on a logarithmic scale. 
(a)



(b)

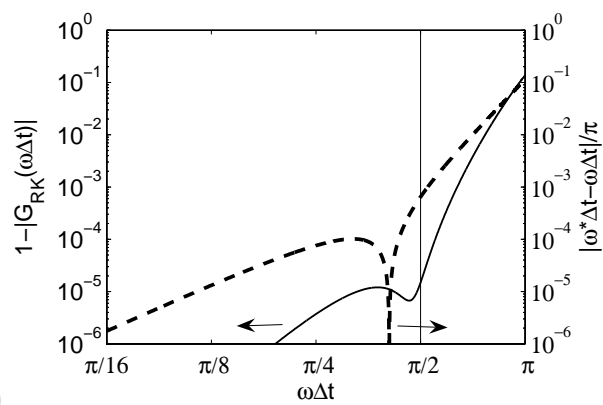

Figure 19: Properties of schemes in the frequency space $\omega \Delta t$. Amplication rate (— ) and phase error ( $-{ }_{-}$) of the optimized Runge-Kutta scheme on linear scale (a) and on logarithmic scale (b). Low-storage fourth-order Runge-Kutta of Bogey and Bailly [38] with 6 sub-steps. 




Figure 20: Initial solution of the 1-D wavepacket on grid 1 ( - ), and on grid 2 $(---)$. 
(a)

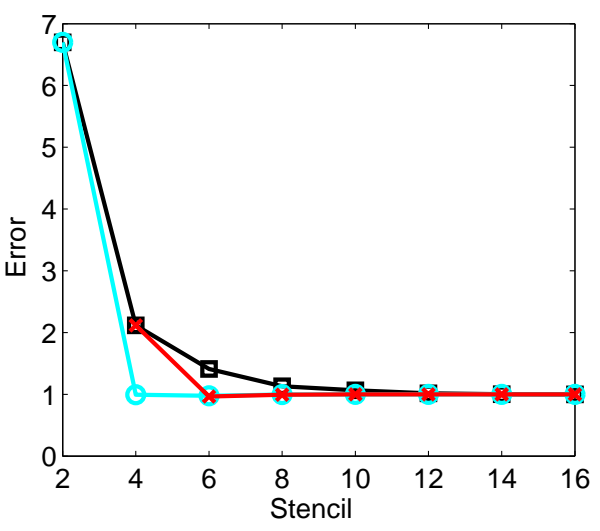

(b)

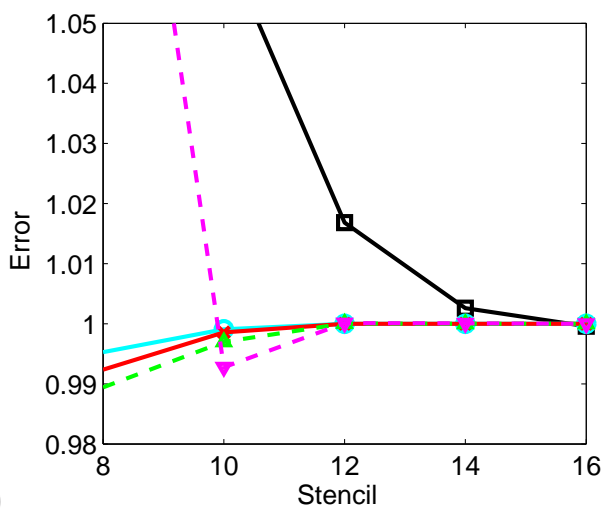

Figure 21: Normalized error at the final iteration for the 1-D advection of a wavepacket as a function of the size $N$ of the interpolation stencil. (a) Lagrange interpolations $(-\square)$, fully optimized interpolations ( $\square$ - ), and optimized interpolation with fourth order constraint $(-\times)$; (b) close-up view for large stencils. Two methods for the evaluation of interpolation coefficient are added: optimized interpolation with sixth-order constraint $(---\triangle)$, and with eighth-order constraint $(---\nabla)$. 

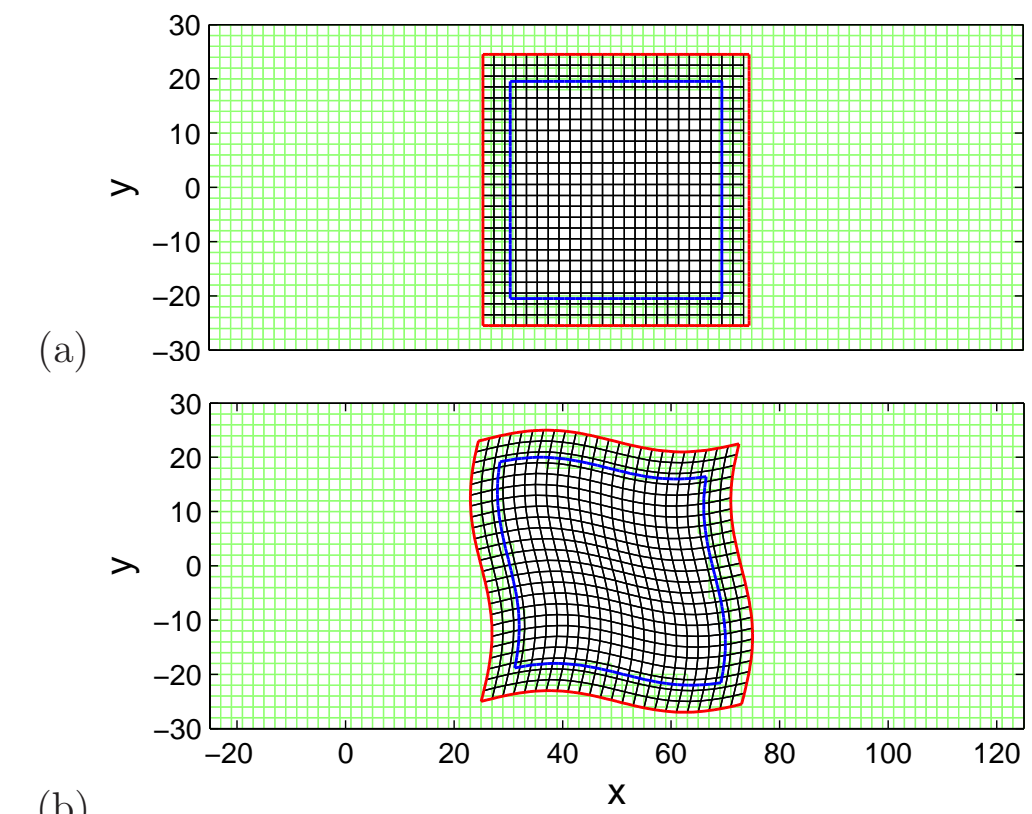

(b)

Figure 22: Overlapping of a Cartesian grid ( - ) by another Cartesian grid (a), or a sinusoidal grid (b) (- ). Every two points are represented. The grid with the higher priority is the foreground grid, so that the cutting hole process is applied to the background grid. 
(a)
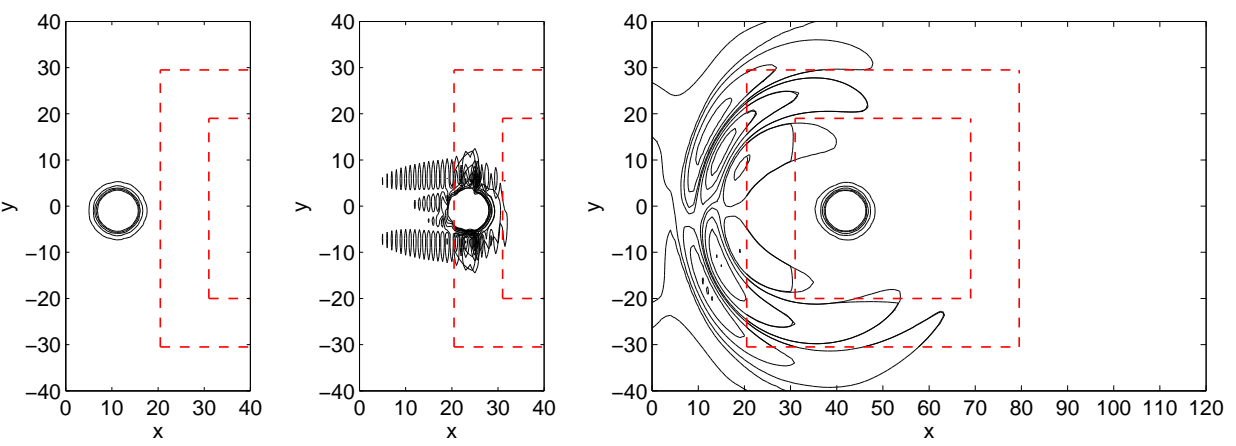

(b)
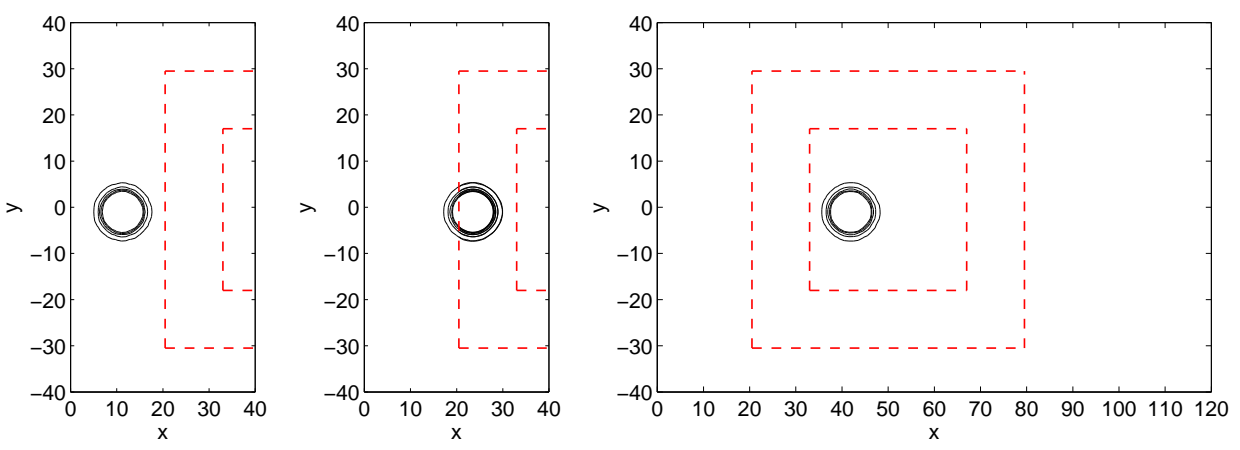

Figure 23: Isocontours of the instantaneous pressure disturbance at three successive instants, $N_{i t}=100,200,350$ (from left to right), showing the passage of the vortex through the first overset interface $(---)$. Lagrange interpolations are used on a $2 \times 2$ (a) and $6 \times 6$ (b) stencil. Isocontours values are $\pm 1 \mathrm{~Pa}$, and \pm 5 to $\pm 20 \mathrm{~Pa}$ with step of $5 \mathrm{~Pa}$. 

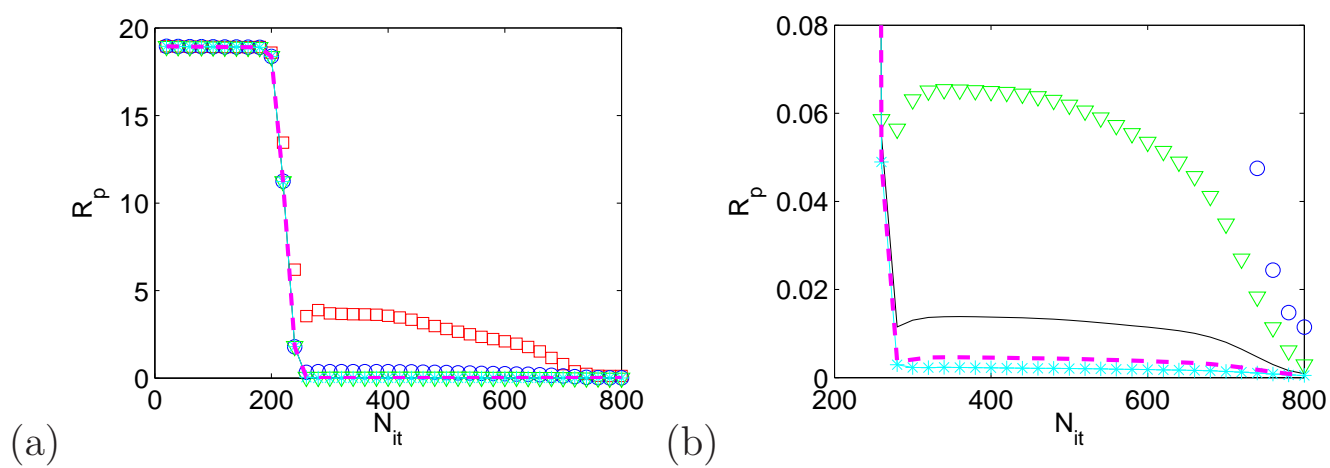

Figure 24: Temporal evolution of the residual pressure $R_{p}$, eq.(35). (a) Stencils $2 \times 2(\square), 4 \times 4(\circ), 6 \times 6(\nabla)$. (b) Close-up view where the results for stencils $8 \times 8(-), 10 \times 10(---), 12 \times 12(-*)$ have been added. The Cartesian foreground grid is used with Lagrange interpolations. 



Figure 25: Advection of a vortex. Normalized error on the pressure between the numerical and analytical solutions at the last iteration with a stencil $N \times N$, for Lagrange interpolations $(-\square)$, fully optimized interpolations $(---\circ)$, and optimized interpolations with $4^{\text {th }}$-order constraint $(-\cdot-\cdot \triangle)$. (a) foreground Cartesian grid, (b) foreground sinusoidal grid. The inserts show close-up for large stencils. For the sinusoidal grid (b), the effect of turning off the high-order evaluation of the offsets with the isoparametric mapping is shown $(\cdots \cdots \nabla)$. 

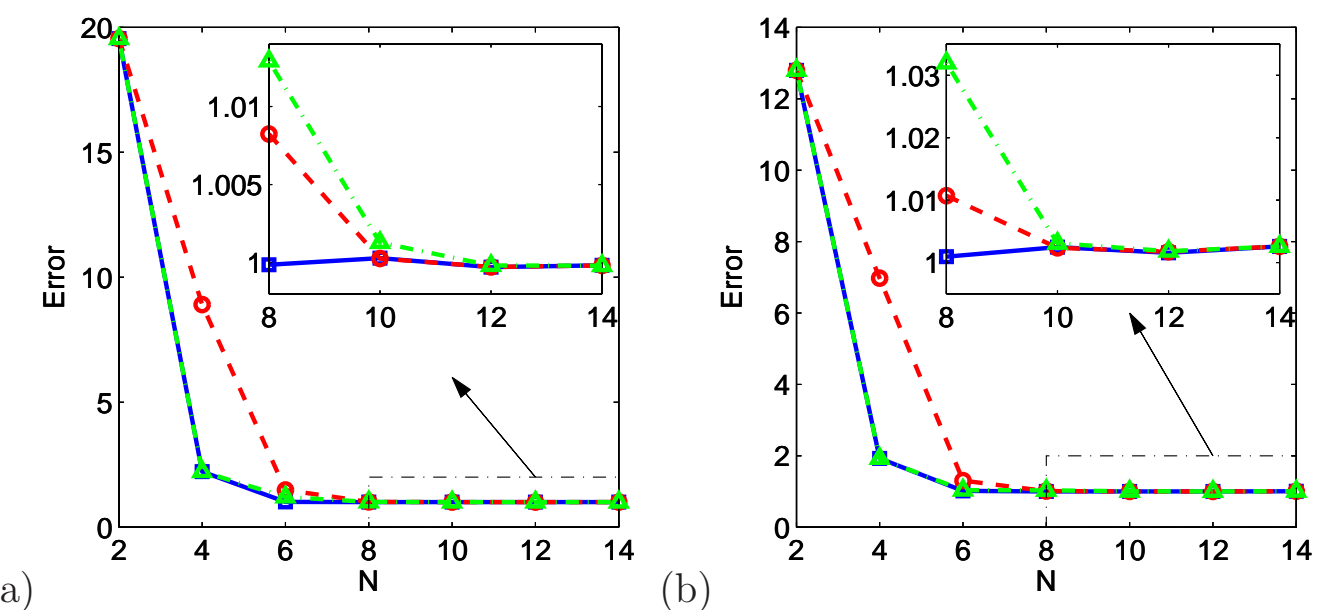

Figure 26: Normalized error for a source of pulsation $\omega=2 \pi / 10 \times c_{\infty} / \Delta x$. Same legend as figure 25: (a) foreground Cartesian grid, (b) foreground sinusoidal grid. 

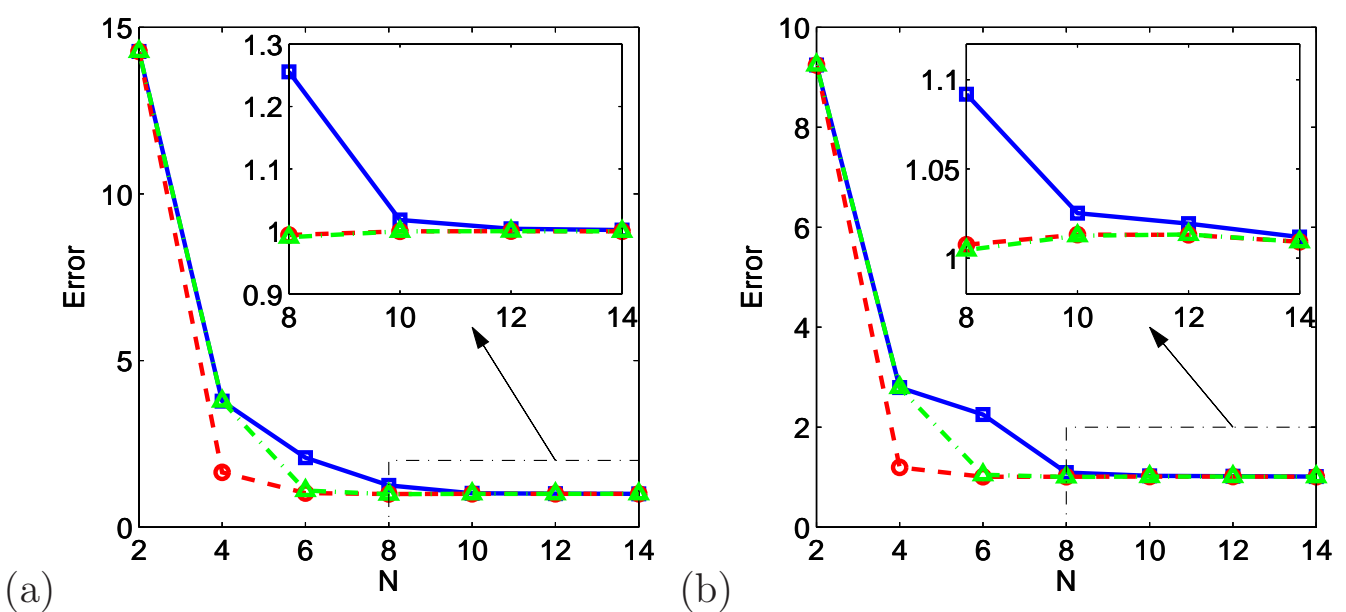

Figure 27: Normalized error for a source of pulsation $\omega=2 \pi / 5 \times c_{\infty} / \Delta x$. Same legend as figure 25: (a) foreground Cartesian grid, (b) foreground sinusoidal grid. 


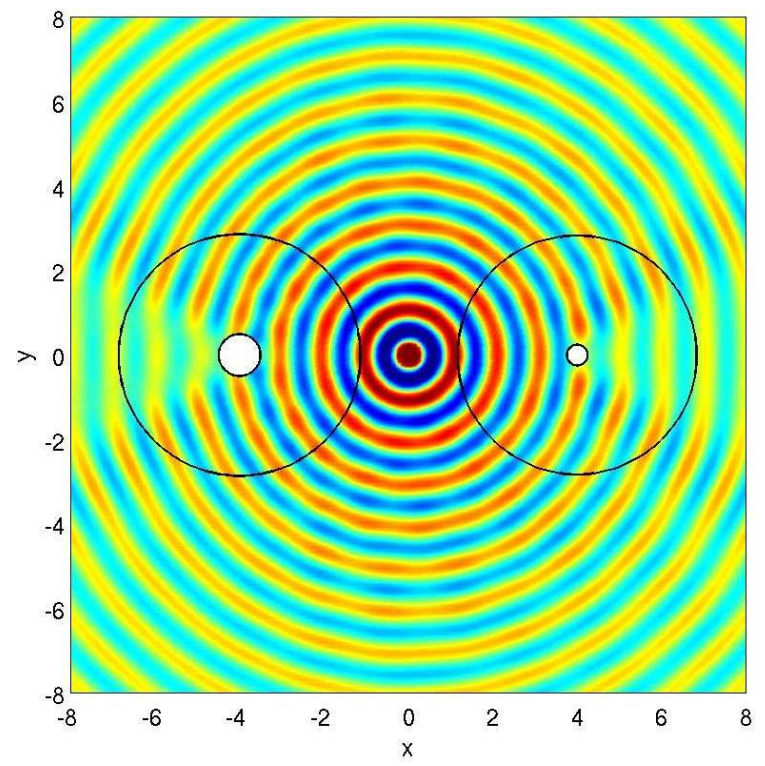

Figure 28: Instantaneous pressure fluctuations. Colormap levels are between - 0.05 $\mathrm{Pa}$ and $0.05 \mathrm{~Pa}$. The black circles indicate the end of the polar grids for each cylinders. 

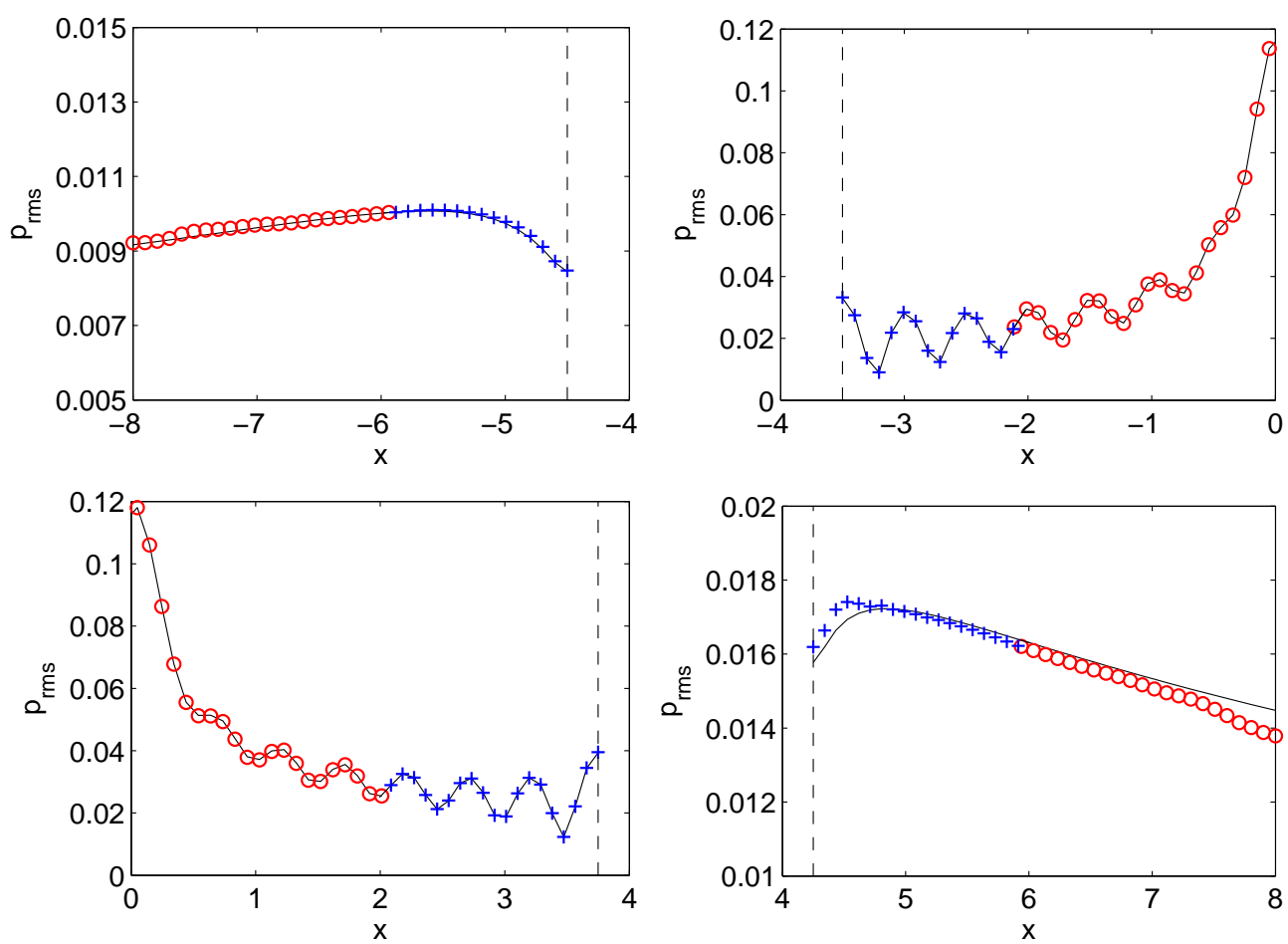

Figure 29: Root mean square pressure along $y=0$ : analytical solution $(-)$, numerical solution for the two polar grids $(+)$ and the Cartesian grid (o). The vertical dashed lines depict the location of the cylinder boundaries. The first two plots are upstream and downstream of the cylinder located at $x=-4 \mathrm{~m}$, and the two last plots show the rms pressure upstream and downstream of the second cylinder at $x=4 \mathrm{~m}$. 




Figure 30: Sketch of the cascade-gust interaction problem. 


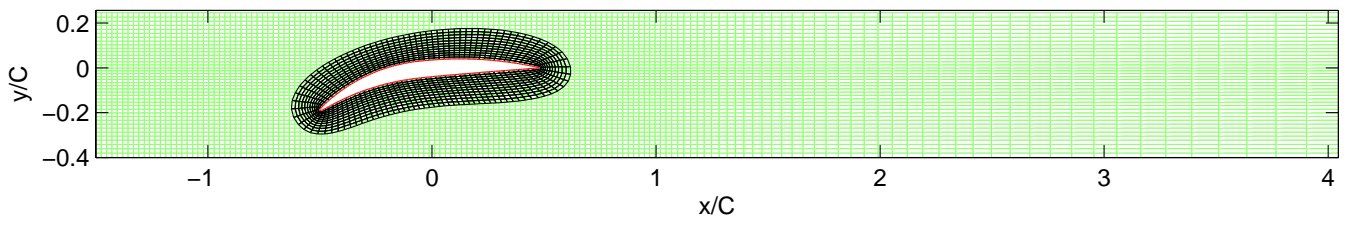

Figure 31: Overlapping grids for one vane passage. Every two grid points are shown. 


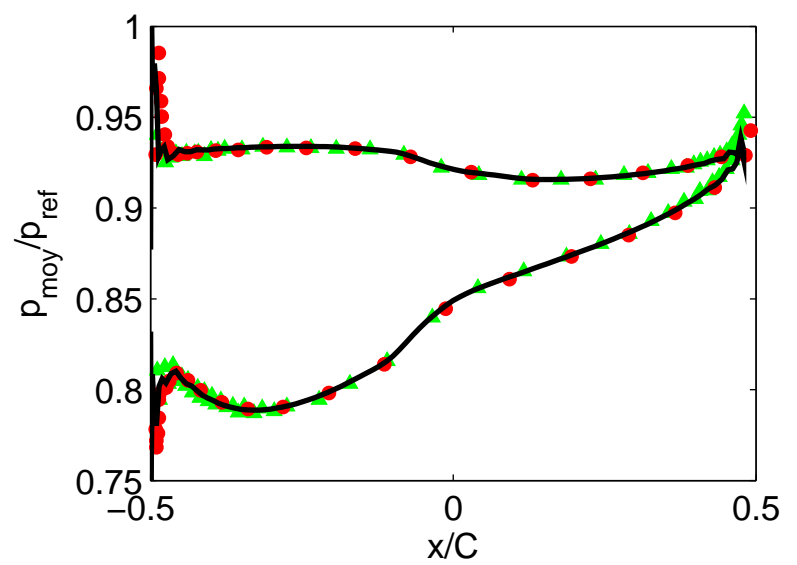

Figure 32: Mean pressure loading on the vane: present overset computation (- - , code Turbo, Envia [45] (•), Nallasamy et al. [43] (ム). 


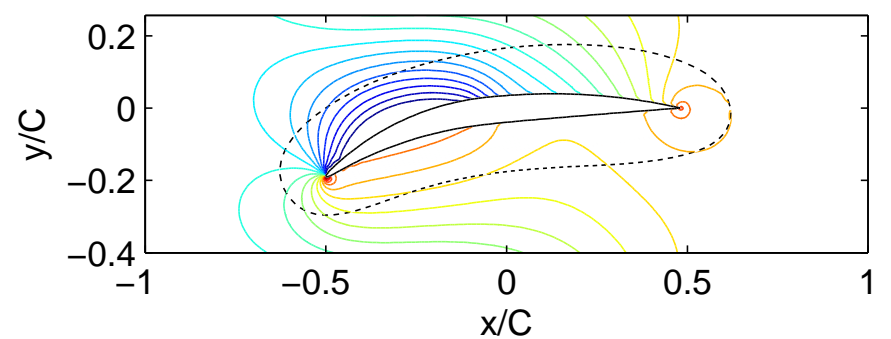

Figure 33: Mean pressure: 20 isocontours equally spaced between 82331 and $98331 \mathrm{~Pa}$. 


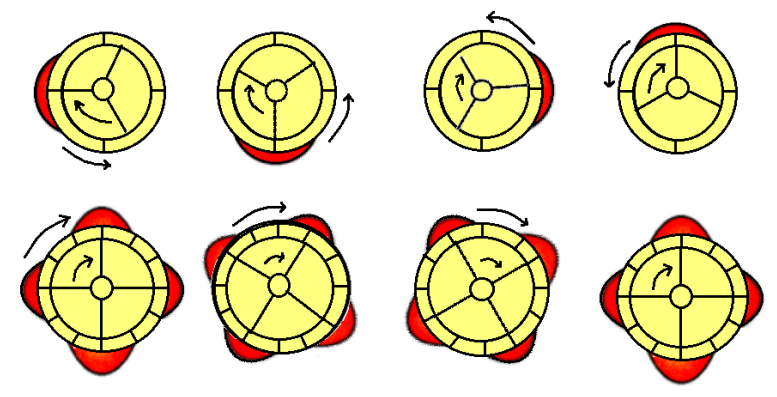

Figure 34: Rotor-Stator interaction with 3 blades and 4 vanes (first row), and 4 blades and 12 vanes (second row). The red lobes mark the acoustic pulses when a blade and a vane are coincident, forming a circumferencial mode. The arrows indicate the direction of rotation of the rotor and of the generated pressure waves. 

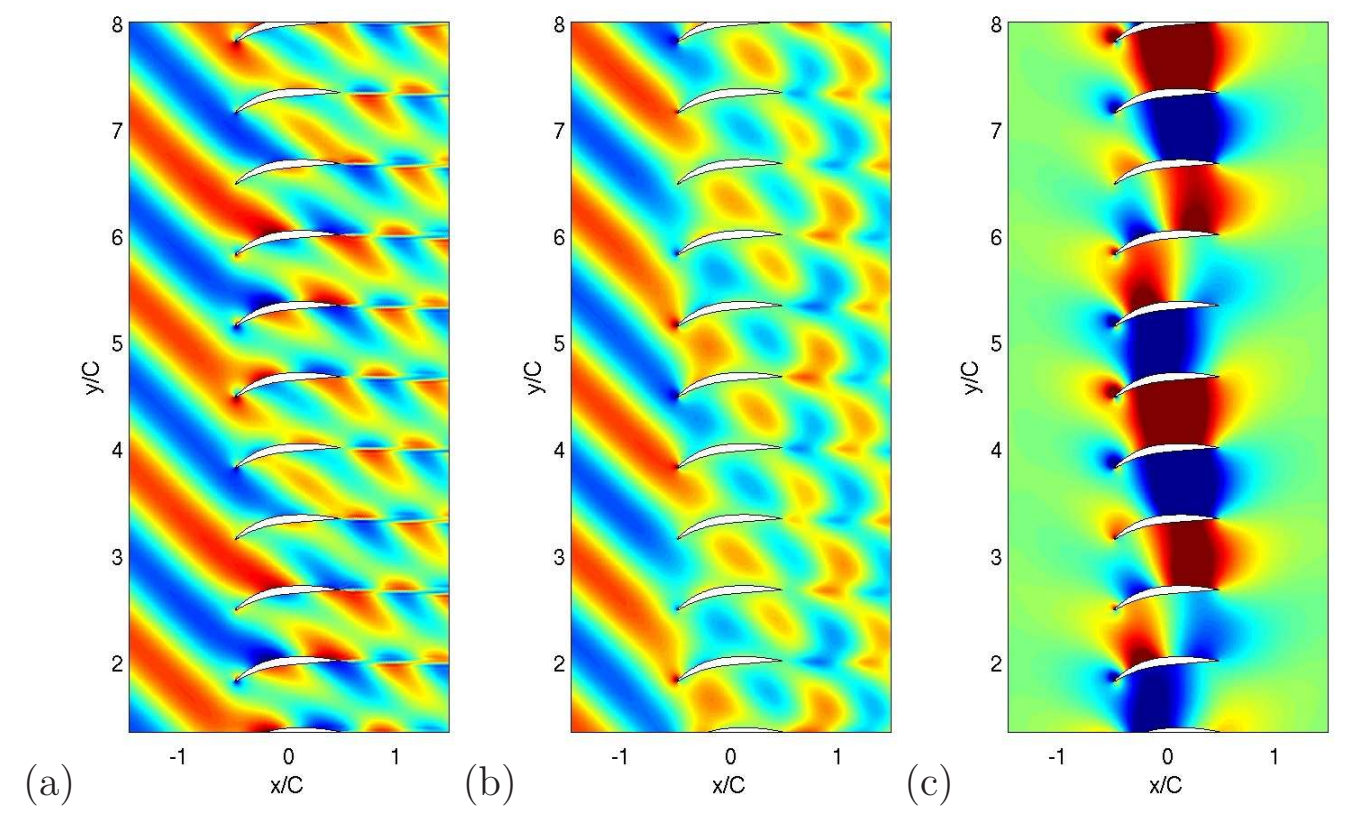

Figure 35: Gust-cascade interaction tone at the first BPF frequency: instantaneous perturbations of the longitudinal velocity $u^{\prime}$ (a), of the transverse velocity $v^{\prime}(\mathrm{b})$, and of the pressure $p^{\prime}$ (c). Colormap bounds are $\pm 2 \mathrm{~m} / \mathrm{s}$ for the velocity maps, and $\pm 200 \mathrm{~Pa}$ for the pressure. 


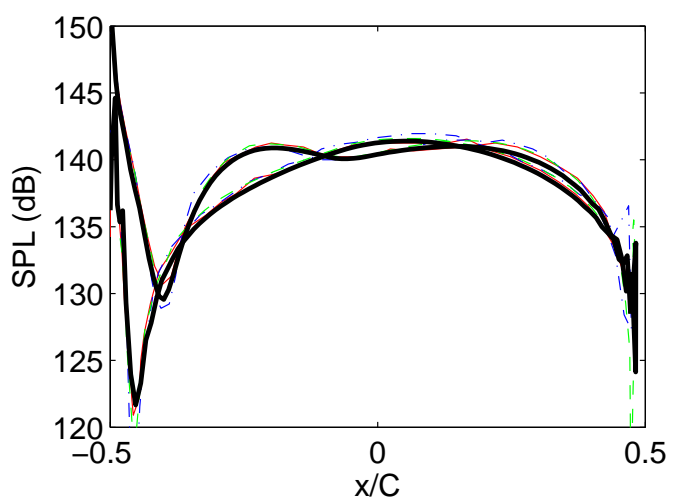

Figure 36: Unsteady pressure distribution over the vane at the first harmonic: (— ) present simulation, (- Coupland [48], (-- ) Nallasamy et al. [43] $(-2.5 \mathrm{~dB}),(-\cdot-\cdot)$ Wang et al. $[49](+4 \mathrm{~dB})$. 

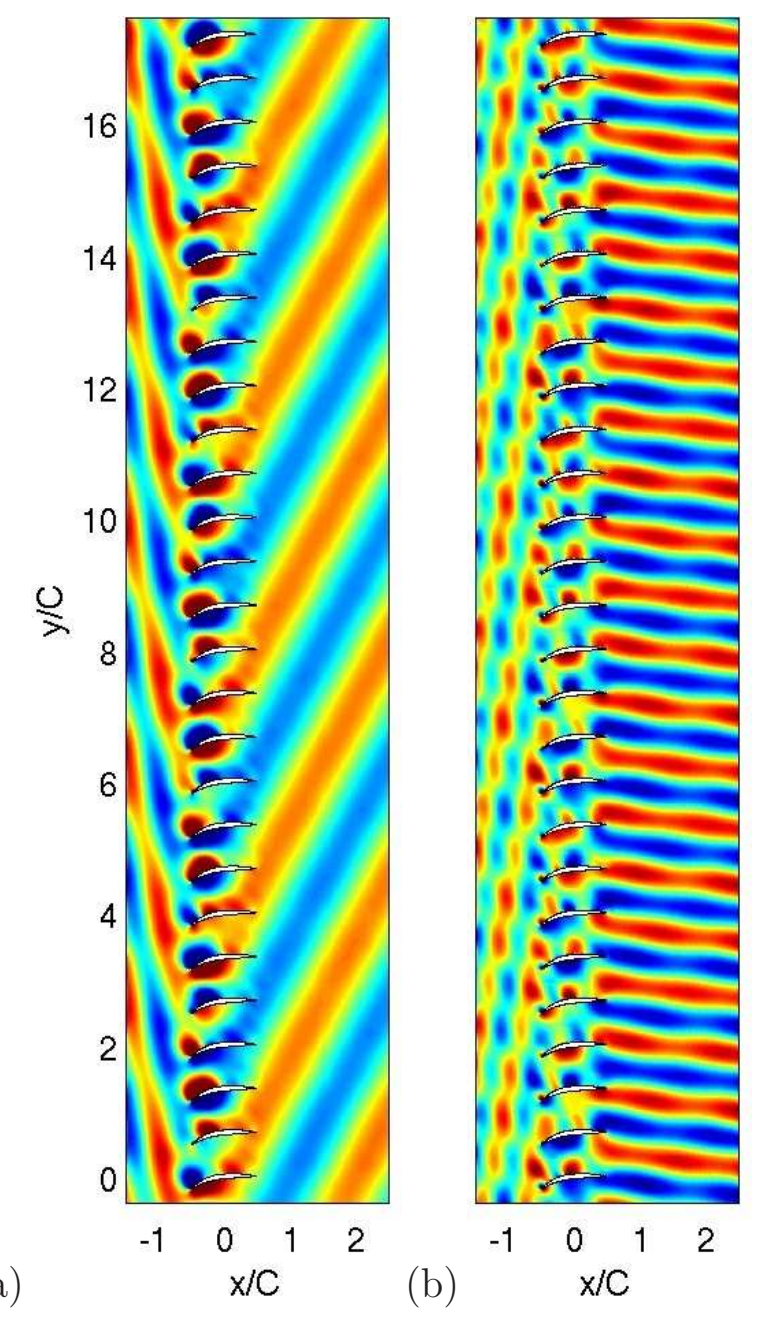

Figure 37: Acoustic rotor-stator tones for the 27 vanes domain. Instantaneous pressure disturbances at the second harmonic (a) with levels $\pm 50 \mathrm{~Pa}$, and at the third harmonic (b) with levels $\pm 12.5 \mathrm{~Pa}$. 


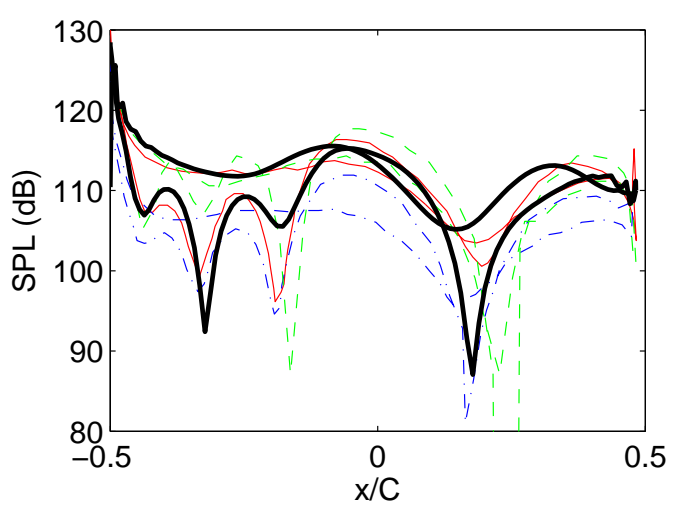

Figure 38: Unsteady pressure distribution over the vane at the third harmonic. Same legend as figure 36 . 


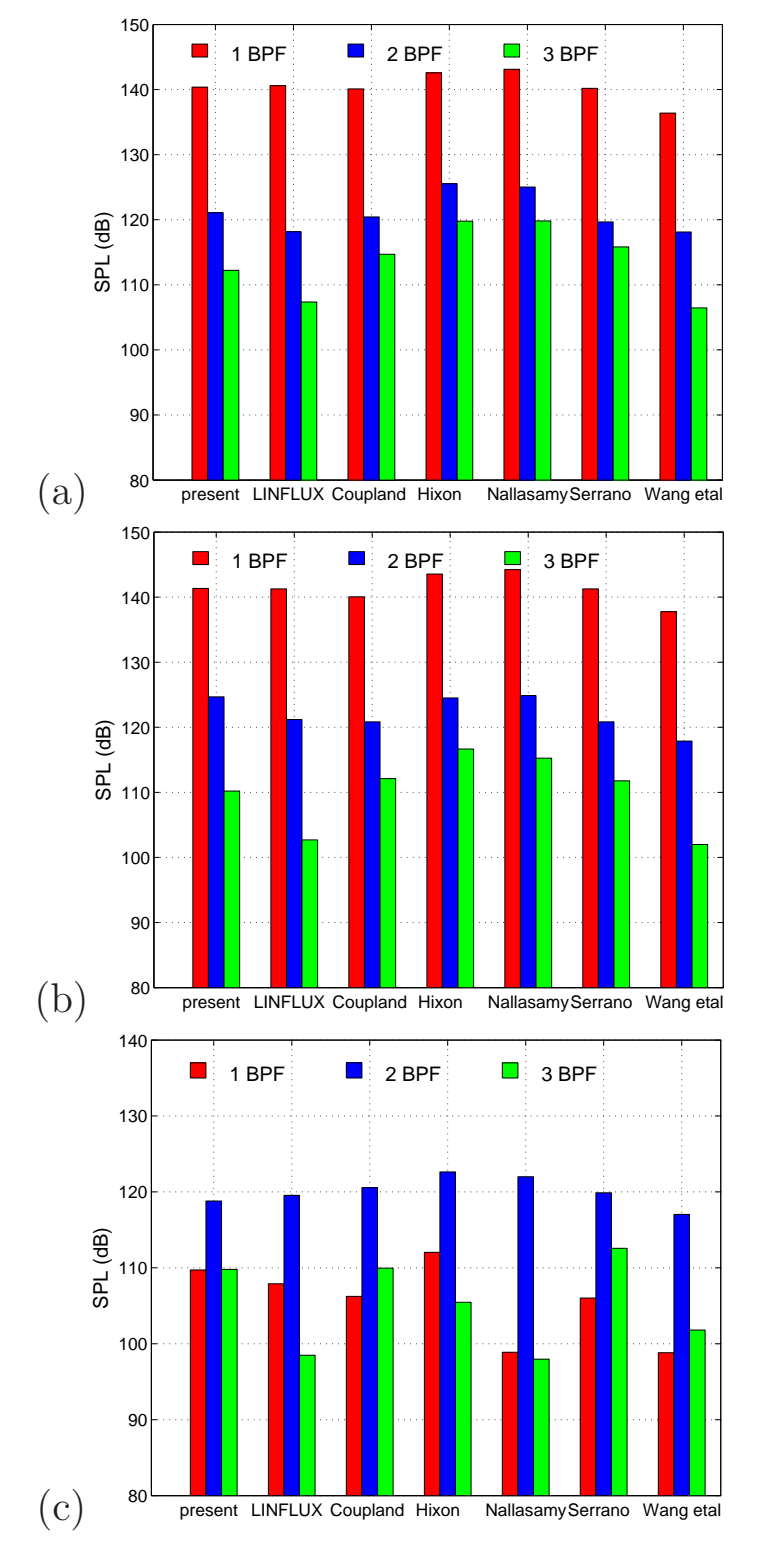

Figure 39: Comparison of overall pressure levels at $x / C=0$ on the suction side (a), on the pressure side (b), and at $y / C=0$ on the outflow plane (c). Values are compared to results of the fourth CAA workshop: Linflux linearized reference by Envia [45], linearized structured solver of Coupland [48], Space-Time Mapping Analysis method of Hixon [51], block-structured Euler solver of Nallasamy et al.[52], unstructured Euler solver of Serrano et al. [46], unstructured CE/SE method by Wang et al. [49]. 